\title{
Scaling of Airplane Dynamic Response to Stochastic Gusts
}

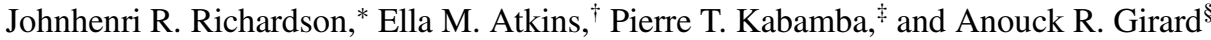 \\ University of Michigan, Ann Arbor, Michigan 48109 \\ DOI: $10.2514 / 1 . C 032410$
}

\begin{abstract}
This paper studies the scaling of airplane dynamic response to stochastic wind gusts, with focus on the relationship between airplane size and airspeed variations. The results have applications in the design and control of small-scale aircraft for robustness with respect to turbulence. Using linearized equations of motion and the Dryden model of gusts, the paper presents analytical and numerical scaling laws for the phugoid natural frequency and damping ratio, and the variances of the airspeed and flight-path angle. The results show that small aircraft are more susceptible to high frequency gusts, that the phugoid damping ratio does not depend directly on airplane size, that the airspeed and flight-path angle variances can be parameterized by the ratio of the phugoid natural frequency to a characteristic turbulence frequency, and that the coefficient of variation of the airspeed decreases with increasing airplane size. Numerical examples validate the results using models of 11 different airplanes, particularly the NASA Generic Transport Model, a hypothetical transport aircraft similar to a Boeing 757, and the NASA T2 subscale jet transport, an operational 5.5\% scale model of the Generic Transport Model.
\end{abstract}

\section{Nomenclature}

$A$

$A_{p}$

$A_{v_{w x}}$

$A_{w}$

$B$

$B_{v_{w x}}$

$C_{D}$

$C_{L}$

$C_{P \mathrm{OL}}$

$C_{v_{w x}}$

$C_{w}$

$C_{w v}$

$c$

$\bar{c}$

$D$

$\mathrm{d}(t)$

E

$E_{p}$

$E_{w}$

$e$

$F_{a}$

$F_{D}$

$F_{g}$

$F_{L}$

longitudinal velocity component

input matrix of Eq. (14)

longitudinal velocity component

drag coefficient

lift coefficient open-loop case

longitudinal velocity component

$\left(\begin{array}{ll}C_{w v}^{T} & C_{w \omega}^{T}\end{array}\right)^{T}$

control-input vector

wing chord length

drives the gusts' coloring filter

Oswald efficiency factor

magnitude of the force of drag

magnitude of the force of lift

bmatrix of $A_{w}$ corresponding to the

state matrix of the gusts' coloring filter

submatrix of $B_{w}$ corresponding to the

output matrix used to compute $P$ in the

submatrix of $C_{w}$ corresponding to the

output matrix of the gusts' coloring filter,

terms of $C_{w}$ that correspond to $\delta v_{w}$

covariance matrix of the noise input $\mathrm{d}(t)$

white noise with distribution $\mathcal{N}(0, D)$ that

disturbance input matrix of Eq. (14)

disturbance input matrix of Eq. (21)

input matrix of the gusts' coloring filter

sum of the forces acting on the airplane that

depend on the relative wind linear and

angular velocities (i.e., aerodynamic forces)

gravitational force acting on the airplane

Received 28 April 2013; revision received 26 November 2013; accepted for publication 3 December 2013; published online 16 April 2014. Copyright (C) 2013 by the authors. Published by the American Institute of Aeronautics and Astronautics, Inc., with permission. Copies of this paper may be made for personal or internal use, on condition that the copier pay the $\$ 10.00$ per-copy fee to the Copyright Clearance Center, Inc., 222 Rosewood Drive, Danvers, MA 01923; include the code 1542-3868/14 and \$10.00 in correspondence with the CCC.

*Ph.D. Candidate, Applied Physics Program, 450 Church Street. Student Member AIAA.

${ }^{\dagger}$ Associate Professor, Department of Aerospace Engineering, 1320 Beal Avenue. Associate Fellow AIAA.

Frofessor, Department of Aerospace Engineering, 1320 Beal Avenue.

${ }^{\S}$ Associate Professor, Department of Aerospace Engineering, 1320 Beal Avenue. Member AIAA.

\begin{tabular}{|c|c|c|}
\hline$F_{0}$ & $=$ & $\begin{array}{l}\text { sum of the forces acting on the airplane that neither } \\
\text { depend on the relative wind nor are gravitational }\end{array}$ \\
\hline$H_{\times}$ & $=$ & skew-symmetric matrix formed from $h$, see Eq. (9) \\
\hline$h$ & & angular momentum of the airplane \\
\hline$I$ & & moment of inertia matrix of the airplane \\
\hline$L_{u}$ & & $\begin{array}{l}\text { characteristic length in the longitudinal turbulence } \\
\text { power spectral density }\end{array}$ \\
\hline$M_{a}$ & $=$ & $\begin{array}{l}\text { sum of the moments acting on the airplane that } \\
\text { depend on the relative wind linear and angular } \\
\text { velocities (i.e., aerodynamic moments) }\end{array}$ \\
\hline$M_{0}$ & $=$ & $\begin{array}{l}\text { sum of the moments acting on the airplane that } \\
\text { do not depend on the relative wind }\end{array}$ \\
\hline$m$ & $=$ & mass of the airplane \\
\hline$N$ & $=$ & $\begin{array}{l}\text { airplane length scale factor, in which the scale } \\
\text { model's length is } N \text { times the full size airplane's }\end{array}$ \\
\hline $\mathcal{N}(\mu, \Sigma)$ & $=$ & $\begin{array}{l}\text { normal distribution with mean } \mu \text { and covariance } \\
\text { matrix } \Sigma\end{array}$ \\
\hline$P$ & $=$ & covariance matrix of $\left(\delta v_{c}^{T} \quad \delta v_{w}^{T}\right)^{T}$ \\
\hline$P_{\mathrm{OL}}$ & $=$ & covariance matrix of the state vector in Eq. (14) \\
\hline$r$ & $=$ & position vector \\
\hline$S$ & $=$ & wing planform area \\
\hline$V$ & $=$ & airspeed, magnitude of $v_{c}$ \\
\hline$V_{c \times}$ & $=$ & skew-symmetric matrix formed from $v_{c}$, see Eq. (9) \\
\hline$v_{c}$ & $=$ & center of mass velocity of the airplane, $\left(\begin{array}{lll}u & v & w\end{array}\right)^{T}$ \\
\hline$v_{w}$ & $=$ & $\begin{array}{l}\text { velocity of the wind relative to the inertial reference } \\
\text { frame, }\left(\begin{array}{lll}v_{w x} & v_{w y} & v_{w z}\end{array}\right)^{T}\end{array}$ \\
\hline$x$ & $=$ & state vector of Eq. (14) \\
\hline$x_{p}$ & $=$ & state vector of Eq. (21) \\
\hline$\gamma$ & $=$ & flight-path angle - \\
\hline$\delta_{a}$ & $=$ & perturbations of the aileron control input \\
\hline$\delta c$ & $=$ & perturbations of $c,\left(\begin{array}{lll}\delta_{a} & \delta_{e} & \delta_{r}\end{array}\right)^{T}$ \\
\hline$\delta_{e}$ & $=$ & perturbations of the elevator control input \\
\hline$\delta_{r}$ & $=$ & perturbations of the rudder control input \\
\hline$\delta v_{c}$ & $=$ & perturbation of $v_{c}$ after perturbing wind \\
\hline$\delta v_{w}$ & $=$ & perturbation of the wind velocity \\
\hline$\delta v_{w x}$ & $=$ & longitudinal component of the wind disturbance \\
\hline$\delta \epsilon$ & $=$ & perturbation of $\epsilon$ after perturbing wind \\
\hline$\delta \omega$ & $=$ & perturbation of $\omega$ after perturbing wind \\
\hline$\delta \omega_{w}$ & $=$ & perturbation of the wind angular velocity \\
\hline $\mathcal{E}$ & $=$ & $\begin{array}{l}\text { nonlinear function expressing } \dot{\epsilon} \text { as a function } \\
\text { of } \omega \text { and } \epsilon\end{array}$ \\
\hline$\epsilon$ & $=$ & $\begin{array}{l}\text { vector comprising the roll and pitch Euler } \\
\text { angles, }(\phi \theta)^{T}\end{array}$ \\
\hline$\zeta_{p}$ & $=$ & damping ratio of the phugoid mode \\
\hline$\kappa$ & $=$ & $\begin{array}{l}\text { relative frequency of the airplane phugoid } \\
\text { mode to the turbulence }\end{array}$ \\
\hline$\xi_{v_{w x}}$ & $=$ & $\begin{array}{l}\text { state variable of the gusts' coloring filter } \\
\text { corresponding to the longitudinal velocity comp }\end{array}$ \\
\hline
\end{tabular}




$\begin{array}{lll}\xi_{w} & = & \text { state vector of the gusts' coloring filter } \\ \rho & = & \text { air density } \\ \rho_{A / C} & = & \text { density of the airplane } \\ \sigma_{u} & = & \text { intensity of the longitudinal turbulence } \\ \mathbf{\Phi} & = & \text { matrix transforming the angular velocity into } \\ & & \text { the Euler rates } \\ \Omega_{\times} & = & \text {skew-symmetric matrix formed from } \omega, \text { see Eq. (9) } \\ \omega & = & \text { angular velocity of the airplane around its center } \\ & & \text { of mass, }(p \quad q \quad r)^{T} \\ \omega_{n p} & = & \text { atural frequency of the phugoid mode } \\ \omega_{w} & = & \text { reference frame, }\left(p_{w} q_{w} r_{w}\right)^{T} \\ \mathbb{1}_{n} & = & \text { identity matrix of dimension } n\end{array}$

T URBULENCE is a common factor in airplane accidents. In a review of over 4000 reports on weather-related accidents between 1992 and 2001, 509, or 12\%, listed weather-related turbulence as a cause or contributing factor [1]. Turbulence contributes to accidents in a variety of ways, including upsets or flight envelope departures that lead to loss of control, substantial damage to aircraft, and injuries to crew and passengers that are sometimes fatal. However, the effects of turbulence on aircraft differ greatly based on aircraft size. This can be seen by normalizing the number of turbulence-related accidents between 1992 and 2001 from the same report [1] by the total number of hours flown by air carriers and general aviation aircraft. Because the 2001 flight hours are readily available for air carriers [2] and general aviation aircraft [3], but the hours for the entire 10-year period are not, 10 times the 2001 flight hours are used for the normalization. Air carriers have a turbulence-related accident rate of $1.25 \times 10^{-5}$ turbulence-related accidents per flight hour, whereas general aviation aircraft and air taxis have a rate of $1.41 \times 10^{-3}$ turbulence-related accidents per flight hour, two orders of magnitude larger. While other factors besides size undoubtedly factor into this much higher turbulence-related accident rate among light aircraft, for example, pilot proficiency and underreporting of general aviation flight hours, this paper shows how aircraft performance might explain this difference.

Figure 1 shows that the airspeed coefficient of variation for an airplane in moderate turbulence decreases with increasing airplane size, measured in this example by wingspan. The coefficient of variation is the standard deviation divided by the mean, a normalized measure of uncertainty in the airspeed. The airplane dynamically scaled for this example is the Lockheed C-5A. Also marked on the figure are the coefficients of variation for 10 other aircraft of various sizes. The figure is described in detail in Sec. V, but the result is intuitive: the fractional change in airspeed due to turbulence is larger for small aircraft.

In addition to general aviation aircraft, a growing fleet of unmanned aircraft systems can benefit from modeling of gust effects on small aircraft. By early 2010, the U.S. military had acquired more than 6100 unmanned aircraft that each weighed $20 \mathrm{lb}$ or less [4]. Moreover, the U.S. military classifies unmanned aerial vehicles over

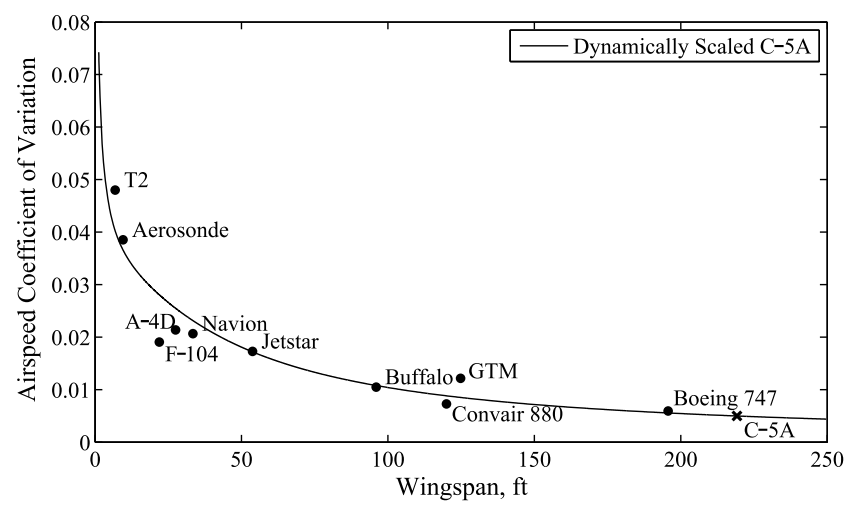

Fig. 1 Dynamically scaled airspeed coefficient of variation vs wingspan.
$5000 \mathrm{lb}$ as large [5], whereas the Federal Aviation Administration's (FAA) wake-turbulence classifications designate airplanes lighter than $41,000 \mathrm{lb}$ as small [6]. Many of these aircraft use autopilots that rely on mathematical models of the airplane's dynamics and performance. The aim of this work was to provide new insight and modeling for airplane dynamic response to stochastic gusts in an effort to make airplanes, especially small airplanes, more robust to turbulence. This aim was accomplished by providing scaling laws for the airplane phugoid mode and for airspeed variations due to gusts.

The present work is a continuation of recent efforts by the authors to analyze the dynamic response of airplanes to turbulence [7-9]. In the prior work [8], the authors present a technique to quantify and visualize the change in airplane performance due to turbulence based on steady flight envelope adjustments. In the present work, the authors analyze how that change in performance scales with airplane size. The modeling required for this analysis primarily draws on two fields: airplane dynamics and wind gust modeling. Detailed discussions of airplane nonlinear and linearized dynamic equations, as well as reduced-order models for these equations, can be found in standard textbooks [10-13]. Some texts also include short sections on modeling gusts $[10,12-14]$. Additional work models the effect of wind gusts $[\underline{15}, \underline{16}]$ and wind shear $[\underline{17}, \underline{18}]$ on aircraft dynamics.

Within the field of flight dynamics, standard scaling laws exist for airplane parameters and performance variables [19-21]. These scaling laws have been used in applications such as system identification of small-scale helicopters [22], development of dynamically scaled aircraft [23], and prediction of the performance of low-Reynolds-number flyers [24]. These scaling laws are derived from the general field of dimensional analysis [25,26], with analogous applications for ships [27], supernovas [28], and in fluid mechanics more generally [29].

Several publications give detailed discussions of wind gust models and their power spectral densities, specifically the Dryden and von Kármán models $[30,31]$. In this literature, it is common to model gusts as stationary random processes defined by given power spectral densities. When modeling wind gusts in this manner, engineers are modeling a type of gust that is continuously varying over long portions of the aircraft's trajectory. Clear air turbulence is a type of gust that can be classified and modeled in this way. Analogous models have been developed for road roughness [32] and rough seas $[33,34]$. The Dryden model has a rational power spectral density, and the von Kármán model has an irrational power spectral density but matches experimental observations more closely than the Dryden model. These two models have standardized forms used by the FAA and the U.S. Department of Defense for design and simulation $[31,35]$. This paper uses these wind models as inputs to the linearized aircraft dynamics to analyze aircraft performance in this type of gust.

In addition to modeling gusts, researchers have also studied the effects of gusts on aircraft. Several good overviews of the field exist $[15,30,36,37]$. Among the results published in this field, Hoblit provides techniques, dimensionless parameters, and examples from a set of charts to characterize uncertainty in the normal load factor due to gust loads using a short period approximation of the linearized airplane dynamic equations [30]. This paper studies uncertainty in the airspeed using a phugoid approximation of the linearized airplane dynamic equations, providing analytical solutions and showing that, in this formulation, one fewer dimensionless parameters are needed to characterize the effects of gusts.

This paper expands upon the scaling laws presented in the literature and applies them to airplane flight through turbulence. Using a phugoid approximation of airplane dynamics, this paper presents three scaling laws: for the phugoid mode, for the airspeed and flight-path angle variances, and for the airspeed coefficient of variation. The phugoid mode analysis shows that smaller airplanes are more sensitive to higher-frequency gusts. The scaling of the airspeed and flight-path angle variances includes analytical solutions for both variances, and shows that they can be parameterized using a new nondimensional parameter: the ratio of the phugoid mode 
natural frequency to the turbulence corner frequency. This nondimensional parameter is itself the ratio of two nondimensional parameters suggested by Hoblit, meaning that this formulation of the gust response requires one fewer nondimensional parameters than the formulation posed by Hoblit [30]. The airspeed coefficient of variation analysis shows that the normalized uncertainty in the airspeed decreases with increasing airplane size, and thus the paper presents a mathematical model showing how smaller airplanes have larger gust response. The paper validates the analytical results using stability derivative models of the NASA Generic Transport Model (GTM) and T2, and compares the results from these two airplanes to a variety of other airplanes.

The paper is divided into seven sections, including the Introduction and Conclusions. Section II summarizes a linearized model for airplane dynamic response to turbulence. Section III summarizes the scaling laws presented in the literature. Section IV summarizes a phugoid approximation of the model in Sec. II, and shows how this phugoid model's natural frequency and damping ratio depend on airplane size. Section IV also derives an analytical solution for the airspeed and flight-path angle variances in the phugoid model in terms of a new nondimensional parameter. Section $\mathrm{V}$ provides numerical examples of the models from Secs. II and IV that dynamically scale a full-size transport airplane, the GTM, in comparison with its scale model, the T2. Section V also shows how the T2 and scaled GTM compare to a variety of other airplanes, and compares different choices of length scale factor. Section VI shows examples of flight envelope reductions due to turbulence for a Navion and an Aerosonde and comments on the applicability of the scaling laws to these envelopes.

\section{Linearized Equations of Motion}

The linearization starts with the nonlinear airplane dynamic equations [11]:

$$
\begin{gathered}
m\left(\dot{v}_{c}+\Omega_{\times} v_{c}\right)=F_{a}\left(v_{c}-v_{w}, \omega-\omega_{w}, \boldsymbol{c}\right)+F_{g}+F_{0} \\
\dot{h}+\Omega_{\times} h=M_{a}\left(v_{c}-v_{w}, \omega-\omega_{w}, \boldsymbol{c}\right)+M_{0} \\
\dot{\epsilon}=\mathcal{E}(\omega, \boldsymbol{\epsilon})
\end{gathered}
$$

in which all the vectors are expressed in the body frame

$$
\begin{gathered}
h=I \omega \\
\mathcal{E}(\omega, \boldsymbol{\epsilon})=\Phi(\boldsymbol{\epsilon}) \omega \\
\Phi(\boldsymbol{\epsilon})=\left(\begin{array}{lll}
1 & \sin \phi \tan \theta & \cos \phi \tan \theta \\
0 & \cos \phi & -\sin \phi
\end{array}\right)
\end{gathered}
$$

and

$$
I=\int_{\text {Volume }} \rho_{A / C}(r)\left(\|r\|^{2} \mathbb{1}_{3}-r r^{T}\right) \mathrm{d} V=\left(\begin{array}{ccc}
I_{x x} & -I_{x y} & -I_{x z} \\
-I_{x y} & I_{y y} & -I_{y z} \\
-I_{x z} & -I_{y z} & I_{z z}
\end{array}\right)
$$

The yaw Euler angle does not affect any of the other variables, so its dynamics are omitted. $\Omega_{\times}$is a skew-symmetric matrix replacing the cross product of a pair of vectors expressed in an orthonormal coordinate system with the inner product of a matrix and a vector, as in the identity:

$$
\boldsymbol{a} \times \boldsymbol{b}=A_{\times} b=B_{\times}^{T} a
$$

$$
A_{\times}(a)=\left(\begin{array}{ccc}
0 & -a_{3} & a_{2} \\
a_{3} & 0 & -a_{1} \\
-a_{2} & a_{1} & 0
\end{array}\right)
$$

The subscript $\times$ indicates that these matrices replaced cross products.

These airplane dynamic equations require a number of assumptions. They assume that Earth is flat and that a point on Earth's surface can be the origin of an inertial reference frame. They assume that the airplane's weight and moment of inertia do not change over time or with altitude. These are standard assumptions justifiable for airplane flight over tens of minutes. They also assume that the airplane is a rigid body, ignoring structural dynamics, also a standard assumption in guidance and control of airplanes.

This paper focuses on the dynamic response of the airplane to wind velocity perturbations. The authors presented in previous work a detailed linearization of the airplane dynamic equations driven by turbulence $[8,9]$. Similar linearizations are available in standard textbooks $[1 \overline{4}]$. After linearizing Eqs. (1-3) with respect to control inputs and wind disturbances and collecting the system of equations into state-space form

$$
\begin{gathered}
\left(\begin{array}{c}
\delta \dot{v}_{c} \\
\delta \dot{\omega} \\
\delta \dot{\epsilon}
\end{array}\right)=\left(\begin{array}{ccc}
-\Omega_{\times}+\frac{1}{m} \frac{\partial F_{a}}{\partial v} & V_{c \times}+\frac{1}{m} \frac{\partial F_{a}}{\partial \omega} & \frac{1}{m} \frac{\partial F_{g}}{\partial \epsilon} \\
I^{-1} \frac{\partial M_{a}}{\partial v} & I^{-1}\left(H_{\times}-\Omega_{\times} I+\frac{\partial M_{a}}{\partial \omega}\right) & 0 \\
0 & \Phi & \frac{\partial \mathcal{E}}{\partial \epsilon}
\end{array}\right)\left(\begin{array}{c}
\delta v_{c} \\
\delta \omega \\
\delta \epsilon
\end{array}\right) \\
+\left(\begin{array}{c}
\frac{1}{m} \frac{\partial F_{a}}{\partial c} \\
I^{-1} \frac{\partial M_{a}}{\partial c} \\
0
\end{array}\right) \delta c-\left(\begin{array}{cc}
\frac{1}{m} \frac{\partial F_{a}}{\partial v} & \frac{1}{m} \frac{\partial F_{a}}{\partial \omega} \\
I^{-1} \frac{\partial M_{a}}{\partial v} & I^{-1} \frac{\partial M_{a}}{\partial \omega} \\
0 & 0
\end{array}\right)\left(\begin{array}{l}
\delta v_{w} \\
\delta \omega_{w}
\end{array}\right)
\end{gathered}
$$

a linear time invariant (LTI) system with control input and wind velocity perturbations as the input, and linear and angular velocity perturbations and Euler angle perturbations as the state. The partial derivatives, which are all of the form of a derivative of a vector with respect to another vector, are used as shorthand for Jacobian matrices. All of the quantities in the state and disturbance matrices, including the derivatives, are evaluated at the steady-flight reference condition around which the aircraft is perturbed.

Note the following about Eq. (10):

1) The wind perturbations are not assumed to be random processes. These equations could also be used to study an airplane's impulse response, the response to pulses approximating discrete gusts, or the step response to wind shear.

2) The forces and moments have not been perturbed with respect to linear or angular acceleration; hence, those effects are assumed to be negligible.

3) To linearize the forces, a small angle of attack is assumed. This assumption is consistent with McClamroch's steady-flight analysis [38]. In the numerical examples of Sec. V, the angle of attack is typically of the order $1 \mathrm{deg}$.

4) Stability-derivative values typically come from wind-tunnel testing and may change with flight state. They are not readily available for many aircraft. Nelson [11] explains how to compute these stability derivatives and tabulates their values for several aircraft.

Through modal analysis, various reduced-order models can be developed for the LTI system in Eq. (10). The most common reducedorder models split the state vector and inputs into longitudinal and lateral components, which are decoupled for many reference conditions. Further reduction to model specific aircraft modes is possible, usually involving changes of variables and ignoring some coupling. The phugoid reduced-order model presented by Stengel [13] is summarized and applied in Sec. IV.

For the wind disturbance in Eq. (10) to represent turbulence, $\delta v_{u}$ and $\delta \omega_{w}$ are modeled as stationary random processes. Such an input is consistent with turbulence found in clear air or storms, but not with wind shear or discrete gusts. Engineers typically use the Dryden and von Kármán models of stochastic gusts [30,31]. Both models define 
gusts in terms of their power spectral densities, and in both cases, the random processes are colored.

Define the spectral density of $\left(\delta v_{w}^{T} \quad \delta \omega_{w}^{T}\right)^{T}$ and assume that it has a rational spectral factorization. This assumption is valid for the Dryden model but can only be approximated for the von Kármán model [30]. Once the spectral density has been factorized, the wind velocity perturbations are modeled as the output of a coloring filter driven by Gaussian white noise $\mathrm{d}(t)$ with zero mean and covariance matrix $D$. Denote a realization of the filter in state-space form as

$$
\begin{gathered}
\dot{\xi}_{w}=A_{w} \xi_{w}+E_{w} \mathrm{~d}(t) \\
\left(\begin{array}{c}
\delta v_{w} \\
\delta \omega_{w}
\end{array}\right)=C_{w} \xi_{w}=\left(\begin{array}{c}
C_{w v} \\
C_{w \omega}
\end{array}\right) \xi_{w} \\
\mathrm{~d}(t) \sim \mathcal{N}(0, D)
\end{gathered}
$$

Examples of such filters, like the one used later from the MATLAB documentation [39], have been derived from the power spectral densities of the Dryden or von Kármán model given in the MIL-STD1797A [31]. The parameters for the power spectral densities and filters depend on the altitude and relative orientation of the airplane with respect to the wind. This paper takes into account the altitude dependence but does not adjust the parameters based on airplane attitude, because all of the examples are for steady level longitudinal flight at small angles of attack and zero sideslip. The MIL-STD1797A gives criteria based on airplane stability derivatives to judge when the angular velocity components of the gusts will be nonnegligible [31].

$$
C_{P \mathrm{OL}}=\left(\begin{array}{cc}
C_{r v} & 0 \\
0 & C_{w v}
\end{array}\right)
$$

The output's covariance matrix is

$$
P=C_{P \mathrm{OL}} \boldsymbol{P}_{\mathrm{OL}} C_{P \mathrm{OL}}^{T}
$$

The authors' previous work [8] shows how to compute the variance of the true airspeed, angle of attack, and normal load factor from this covariance matrix. It also shows how to compute safety margins and flight envelopes for steady-flight maneuvers performed in turbulence based on the covariance matrix. This paper frames results in terms of the variance of the true airspeed $V$. Because the paper assumes the reference wind velocity is zero, the true airspeed is also the magnitude of the center of mass velocity $v_{c}$.

Solving the full Lyapunov equation, Eq. (16), analytically is cumbersome. Numerical solutions require $\mathcal{O}\left(n^{2}\right)$ memory and $\mathcal{O}\left(n^{3}\right)$ computations, in which $n$ is the dimension of the state matrix [40]. In later sections, analytical solutions are considered for an analogous Lyapunov equation derived for a reduced-order model of these dynamics.

\section{Dynamic Scaling}

To compare aircraft of different sizes, the aircraft should be dynamically scaled according to established similitude requirements and scaling laws. Wolowicz et al. [20] present a comprehensive review of similitude requirements for scale models of aircraft. Their review describes similarity of geometry, angle of attack, Reynolds number, Froude number, and Mach number, and lists other

Appending the gust filter to Eq. (10)

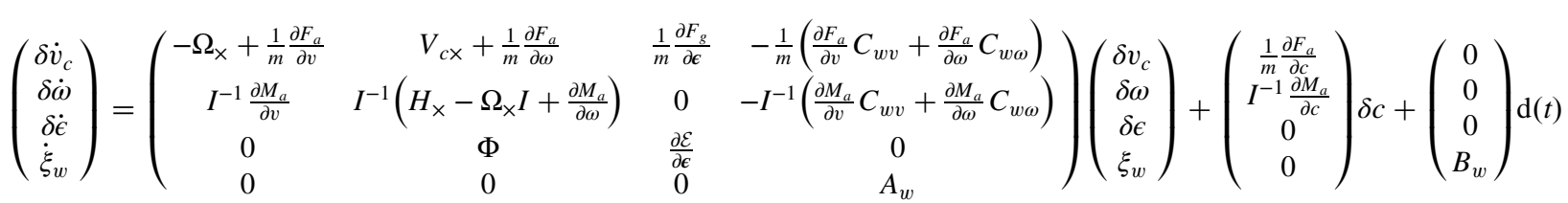

or more compactly

$$
\dot{x}=A x+B \delta c+E d(t)
$$

The system given by Eq. (14) is linear and driven by zero-mean, Gaussian white noise. In applications with asymptotically stable dynamics, the perturbations $\delta v_{c}$ and $\delta \omega$ are also zero-mean, Gaussian, stationary random processes. For a white noise input to Eq. (14), the steady-state covariance $\boldsymbol{P}_{\mathrm{OL}}$ of this open-loop system's state is given by the solution of the Lyapunov equation:

$$
A \boldsymbol{P}_{\mathrm{OL}}+\boldsymbol{P}_{\mathrm{OL}} A^{T}+E D E^{T}=0
$$

For $\boldsymbol{P}_{\mathrm{OL}}$ to be finite, unique, and positive definite, two conditions must hold. First, $A$ must be asymptotically stable. Second, $\left(A, E_{c}\right)$ must be controllable, in which, because $E D E^{T}$ is positive semidefinite, it can be factorized as $E_{c} E_{c}^{T}$. Both of these conditions hold in the GTM and T2 examples of Sec. V. For cases with unstable dynamics, feedback control can be implemented, and a Lyapunov equation can be formed for the closed-loop system [8].

For the applications considered later, only the covariance of the airplane and wind linear velocities matter. Thus, choose $\left(\begin{array}{ll}\delta v_{c}^{T} & \delta v_{w}^{T}\end{array}\right)^{T}$ as the output of the LTI system, corresponding to the output matrix: similarities that may be important in applications. For geometric similarity, a set of power law relations serves as scaling laws [19,22]:

$$
\text { Length: } l_{m}=N l_{f}
$$

$$
\text { Area: } S_{m}=N^{2} S_{f}
$$

$$
\text { Mass: } m_{m}=N^{3} m_{f}
$$

$$
\text { Inertia: } I_{m}=N^{5} I_{f}
$$

$$
\text { Speed: } v_{m}=\sqrt{N} v_{f}
$$

$$
\text { Angular rate: } \omega_{m}=\frac{1}{\sqrt{N}} \omega_{f}
$$

in which the subscripts $m$ and $f$ stand for model and full size, and $N$ is the length scale factor, meaning a quantity such as the model aircraft's wingspan will be $N$ times the wingspan of the full-size aircraft. 
In the work of Burk and Wilson [19] and Jordan et al. [23,41], scaling is used to design small-scale model airplanes upon which experiments are conducted that give insight into the performance of the full-size airplane. On the other hand, in Shyy et al. [24] and Tennekes [42], scaling laws presented at the beginning of the book are meant to explain how parameters and performance variables can be expected to vary over aircraft and birds of different sizes. This paper uses scaling in a manner similar to Shyy et al. [24] and Tennekes [42], although birds are not considered. The contributions of this paper center on deriving other scaling laws from those mentioned previously and drawing conclusions about how aircraft performance is a function of aircraft size. As such, the scale factor $N$ is often referred to in this paper as a measure of aircraft size relative to some arbitrary baseline aircraft.

The scaling relationships mentioned previously also depend on the density of the air, because, in many cases, a model and full-size aircraft do not operate at the same altitude. Rather than include the density explicitly in the scaling laws mentioned previously, this paper assumes that smaller aircraft operate at lower altitudes than their larger counterparts, or

$$
\rho=\rho(a(N)), \quad \frac{\mathrm{d} \rho}{\mathrm{d} N}<0
$$

where here, $a$ is the altitude. Equation (20) says that the air density decreases with increasing $N$ over the range of length scales for fixed wing flight. In the figures in Sec. V, however, the air density is held constant for the dynamic scaling, that is, each curve compares scaled aircraft at a particular altitude. On a related note, the turbulence parameters in the Dryden model vary with altitude. For fixed probability of exceedance, the most severe turbulence generally occurs at roughly $5000 \mathrm{ft}$ above the ground [31].

Note that some of the models of aerodynamic forces and moments in airplane flight assume a large Reynolds number. As the length scale becomes sufficiently small, this assumption ceases to hold. This paper only considers length scales relevant for fixed wing flight at high Reynolds number. A detailed analysis of aerodynamics at Reynolds numbers of $10^{5}$ and below is available in Shyy et al. [24].

\section{Turbulence in the Phugoid Model}

\section{A. Phugoid Model}

The phugoid model described by Stengel [13] is a model order reduction that captures the airspeed and flight-path angle dynamics of the linearized dynamics presented in Sec. II. As described in detail in the authors' previous work, the airspeed statistics can be used to compute safety margins and envelopes for steady-flight maneuvers performed in turbulence [8], hence the interest in the phugoid approximation. In particular, the airspeed statistics can be used to identify steady-flight states close to the stall and propulsion boundaries of the steady flight envelope, including the flight ceiling, that are unlikely to be maintainable and prone to loss of control in turbulence. The phugoid equations of motion are

$$
\left(\begin{array}{c}
\delta \dot{V} \\
\delta \dot{\gamma}
\end{array}\right)=\left(\begin{array}{cc}
-\frac{1}{m} \frac{\partial F_{D}}{\partial V} & -g \cos \gamma \\
\frac{1}{m V} \frac{\partial F_{L}}{\partial V} & \frac{g \sin \gamma}{V}
\end{array}\right)\left(\begin{array}{c}
\delta V \\
\delta \gamma
\end{array}\right)+\frac{1}{m}\left(\begin{array}{c}
\frac{\partial F_{D}}{\partial V} \\
-\frac{1}{V} \frac{\partial F_{L}}{\partial V}
\end{array}\right) \delta v_{w x}
$$

or more compactly

$$
\delta \dot{x}_{p}=A_{p} \delta x_{p}+E_{p} \delta v_{w x}
$$

From here, the discussion is limited to level reference conditions (i.e., $\gamma=0)$. The characteristic equation for this system is

$$
s^{2}+\frac{1}{m} \frac{\partial F_{D}}{\partial V} s+\frac{g}{m V} \frac{\partial F_{L}}{\partial V}=0
$$

From the coefficients of the characteristic equation, the natural frequency and damping ratio are

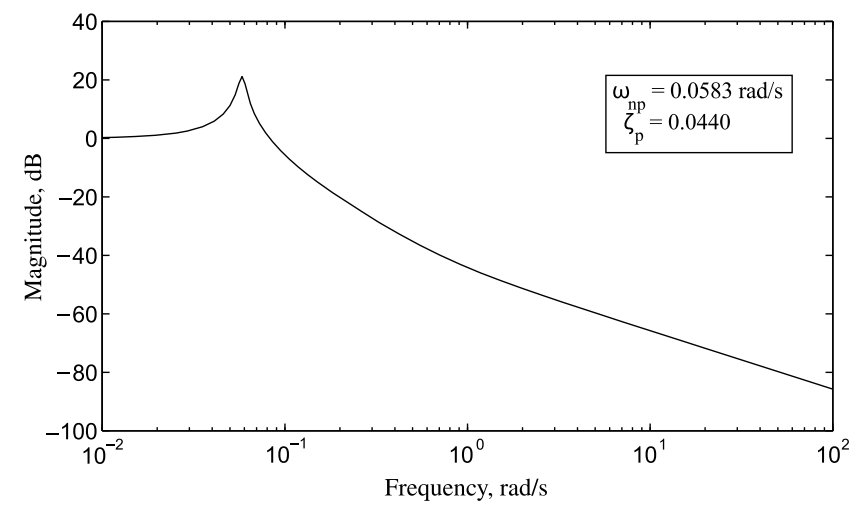

Fig. 2 Bode magnitude plot for the GTM.

$$
\begin{aligned}
& \omega_{n p}=\sqrt{\frac{g}{m V} \frac{\partial F_{L}}{\partial V}} \\
& \zeta_{p}=\frac{1}{2 m \omega_{n p}} \frac{\partial F_{D}}{\partial V}
\end{aligned}
$$

Qualitatively, the frequency response of both the airspeed and the flight-path angle to a wind disturbance has a flat or upward sloping low frequency response, a resonant peak at $\omega_{n p}$ if $\zeta_{p}<0$, and a high frequency response that falls off with frequency. Figure 2 shows the Bode magnitude plot for the GTM's phugoid approximation.

For the forces of lift and drag

$$
\begin{gathered}
F_{L}=\frac{1}{2} \rho S C_{L} V^{2} \\
F_{D}=\frac{1}{2} \rho S C_{D} V^{2}
\end{gathered}
$$

$$
\begin{aligned}
& \frac{\partial F_{L}}{\partial V}=\rho S C_{L} V+\frac{1}{2} \rho S V^{2} \frac{\partial C_{L}}{\partial V} \\
& \frac{\partial F_{D}}{\partial V}=\rho S C_{D} V+\frac{1}{2} \rho S V^{2} \frac{\partial C_{D}}{\partial V}
\end{aligned}
$$

Set $\partial C_{L} / \partial V=\partial C_{D} / \partial V=0$ for subsonic flight, as it is, for example, in all of the stability derivative models presented in the appendix of Nelson [11]. Substituting these expressions into the natural frequency and damping ratio

$$
\begin{gathered}
\omega_{n p}=\sqrt{\frac{g \rho S C_{L}}{m}} \\
\zeta_{p}=\frac{C_{D} V}{2} \sqrt{\frac{\rho S}{m g C_{L}}}
\end{gathered}
$$

Substituting the scaling laws in Eqs. (19a-19f) gives a scaling law for the phugoid mode.

Scaling Law 1: Phugoid Mode

$$
\omega_{n p} \propto \sqrt{\frac{\rho(N)}{N}}
$$

$$
\zeta_{p} \propto \sqrt{\rho(N)}
$$


The natural frequency decreases with increasing airplane size. Its $N^{-1 / 2}$ dependence is consistent with the angular rate scaling law of Eq. (19f). It has an additional dependence through the air density that also decreases with increasing size. The damping ratio decreases with increasing $N$, but only due to the higher flight altitude of larger aircraft. Because of these relationships, small aircraft are more sensitive to high frequency gusts than large aircraft. Similarly, the resonant peak of small aircraft is at a higher frequency than that of large aircraft and is smaller due to increased air density at low altitude.

\section{B. Covariance of the Phugoid Model}

The phugoid model is driven by gusts along the same direction as the velocity vector. At altitudes above $2000 \mathrm{ft}$, the Dryden turbulence model provides the power spectral density and coloring filter transfer function for turbulence along the aircraft longitudinal axis [39]. For small angles of attack and sideslip, the gust components along the wind and body frame $x$ axes are approximately equal. Appending a state variable of the coloring filter to Eq. (22)

$$
\left(\begin{array}{l}
\delta \dot{x}_{p} \\
\dot{\xi}_{v_{w x}}
\end{array}\right)=\left(\begin{array}{cc}
A_{p} & E_{p} C_{v_{w x}} \\
0 & A_{v_{w x}}
\end{array}\right)\left(\begin{array}{l}
\delta x_{p} \\
\xi_{v_{w x}}
\end{array}\right)+\left(\begin{array}{c}
0 \\
B_{x w}
\end{array}\right) n(t)
$$

As with Eq. (16), a Lyapunov equation can provide this LTI system's state variables' covariance. Solving the Lyapunov equation analytically and substituting in Eqs. (28) and (29), as well as the coloring filter matrices, yield analytical expressions for the airspeed and flight-path angle variances. Relating them to the scaling laws already presented leads to a scaling law for these variances.

Scaling Law 2: Airspeed and Flight-Path-Angle Variances

$$
\begin{gathered}
\sigma_{V}^{2}=\left(\frac{\sigma_{u}^{2}}{\pi}\right) \frac{2 \zeta_{p} \kappa+\kappa / 2 \zeta_{p}+\kappa^{2}}{1+2 \zeta_{p} \kappa+\kappa^{2}} \\
\sigma_{\gamma}^{2}=\left(\frac{\sigma_{u}^{2}}{V^{2} \pi}\right)\left(\frac{C_{L}}{C_{D}}\right)^{2} \frac{2 \zeta_{p} \kappa}{1+2 \zeta_{p} \kappa+\kappa^{2}} \\
\kappa \triangleq \frac{\omega_{n p}}{\omega_{\text {turb }}} \\
\omega_{\text {turb }} \triangleq \frac{V}{L_{u}}
\end{gathered}
$$

After substituting in the scaling laws for $\omega_{n p}$ and $V$

$$
\kappa \propto \frac{1}{N}
$$

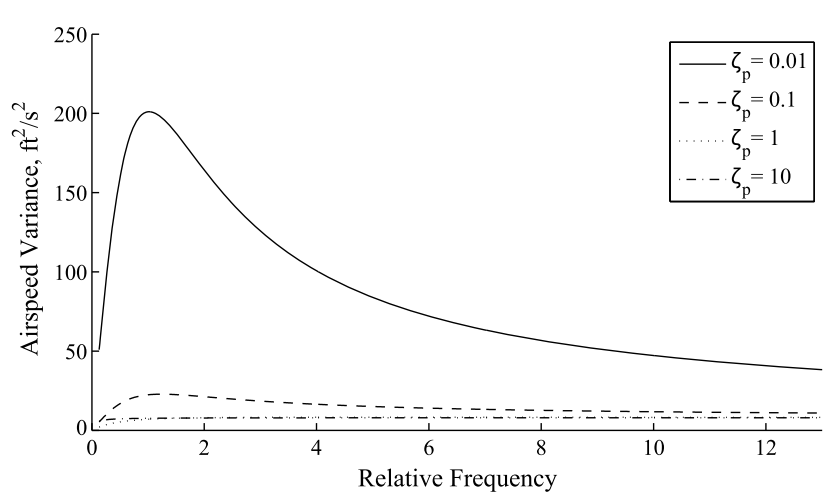

Fig. 3 Airspeed variance vs relative frequency of phugoid mode to turbulence at different phugoid damping ratios.
The frequency $\omega_{\text {turb }}$ is the corner frequency of the longitudinal turbulence power spectral density. Thus, $\kappa$ is the relative frequency of the airplane phugoid mode to the turbulence corner frequency. To understand why the turbulence's corner frequency depends on the airplane's airspeed, recall that the Dryden and von Kármán models assume a spatially varying turbulence velocity field frozen in time. The temporal frequency content of the stochastic gusts depends on how fast the airplane travels through the spatially varying field.

Figures $\underline{3}$ and $\underline{4}$ show how $\sigma_{V}^{2}$ and $\sigma_{\gamma}^{2}$ depend on $\kappa$ and $\zeta_{p}$. The other parameters, namely $C_{L} / C_{D}, \sigma_{u}^{2}$, and $\sigma_{u}^{2} / V^{2}$, are kept fixed at the GTM's values for steady level longitudinal flight at Mach 0.8 and $35,000 \mathrm{ft}$ in moderate turbulence. The airplane and turbulence model parameters are described in detail in Sec. $\underline{V}$ and Appendix $\underline{A}$. The range of values for $\kappa$ starts at the GTM's value at the small end and increases until the length scale factor $N=0.01$ in Eq. (39). Both $\sigma_{V}^{2}$ and $\sigma_{\gamma}^{2}$ have peaks near the low end of this range of $\kappa$. The range of values for $\zeta_{p}$ starts at the order of magnitude of the GTM and T2 and goes up three orders of magnitude. This range of $\zeta_{p}$ allows us to speculate about the effects on the variances of feedback control to improve phugoid damping. Increasing $\zeta_{p}$ causes a decrease in $\sigma_{V}^{2}$, except for combinations of low values of $\kappa$ and high values of $\zeta_{p}$. It also causes an increase in $\sigma_{\gamma}^{2}$.

To investigate the peaks of both variances with respect to $\kappa$, take the derivatives of $\sigma_{V}^{2}$ and $\sigma_{\gamma}^{2}$ with respect to $\kappa$, and set them equal to zero. The peaks of $\sigma_{V}^{2}$ and $\sigma_{\gamma}^{2}$ occur, respectively, at

$$
\begin{gathered}
\kappa=2 \zeta_{p}+\sqrt{1+8 \zeta_{p}^{2}} \\
\kappa=1
\end{gathered}
$$

The peaks indicate resonance between the turbulence and the aircraft taking place at these relative frequencies. For the airspeed variance, that resonance depends on the phugoid damping, but for the flightpath angle variance, the phugoid damping does not matter.

\section{Comparison with Hoblit Parameters}

In work analogous to the preceding results, Hoblit studies the uncertainty in the normal load factor due to gust loads using a short period approximation to the linearized airplane dynamics [30]. Hoblit focuses on the root-mean-square normal load factor in the short period approximation instead of the variance of the airspeed and flight-path angle in the phugoid approximation. He identifies four dimensionless parameters that characterize the response. In this paper, the authors call them

$$
\frac{\delta_{\mathrm{sp}}}{L_{w}}, \quad \frac{\bar{c}}{\delta_{\mathrm{sp}}}, \quad \frac{\omega_{\mathrm{sp}} \delta_{\mathrm{sp}}}{v_{t}}, \quad \zeta_{\mathrm{sp}}
$$

in which $L_{w}$ is the characteristic length of vertical gusts, $\bar{c}$ is the airplane chord length, $f_{\mathrm{sp}}$ is the short period natural frequency, $\zeta_{\mathrm{sp}}$ is the short period damping ratio, and

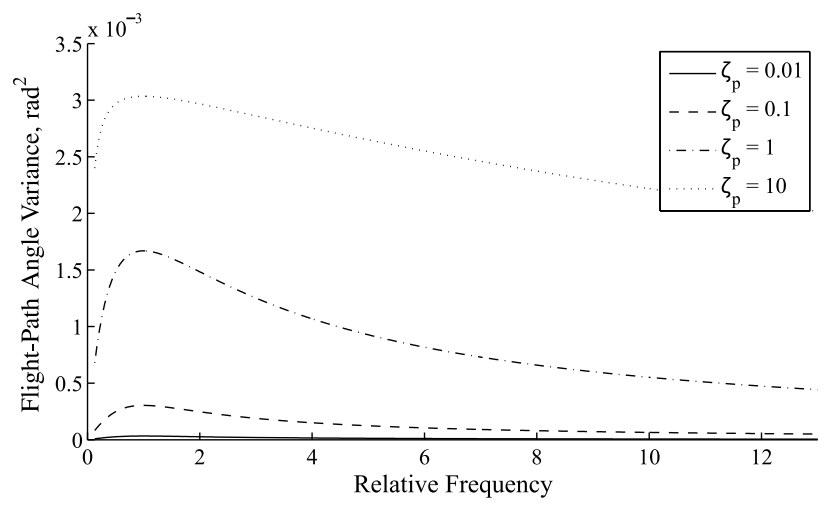

Fig. 4 Flight path angle variance vs relative frequency of phugoid mode to turbulence at different phugoid damping ratios. 


$$
\delta_{\mathrm{sp}} \triangleq \frac{2 m}{\rho S C_{L_{\alpha}}}
$$

a characteristic airplane length.

Hoblit's dimensionless parameter $\bar{c} / \delta_{\mathrm{sp}}$ describes unsteady effects [30]. This paper does not study its effect in the phugoid approximation, although Table B1 in Appendix B quotes its value for the various airplanes used in the upcoming examples to show that unsteady effects should be small. Equations (35) and (36) show that, as in the short period approximation, the phugoid damping ratio can be used as one of the dimensionless parameters to describe the response. As for Hoblit's other two dimensionless parameters, note that dividing them gives the dimensionless parameter $f_{\mathrm{sp}} L_{w} / v_{t}$, a short period analog of $\kappa$ defined in Eq. (37). Thus, in this paper's formulation of the gust response, these two dimensionless parameters of Hoblit only appear as a ratio, reducing the number of dimensionless parameters needed to characterize the response. As described by Szirtes and Rózsa, the number of curves and charts necessary to characterize a dependent variable in terms of a set of independent variables, not to mention the effort that goes into creating and using such charts, grows exponentially with the number of independent variables [26]. Thus, a reduction from four dimensionless parameters to three is a marked improvement.

\section{Comparison of Scaled Airplane Dynamic Response}

This section gives numerical examples of the results from the previous section. The numerical examples are based on the Dryden turbulence model and stability derivative models of two NASA airplanes. Some of the examples also include nine other airplanes. Appendix A discusses the parameters for the airplanes and the turbulence.

The first NASA airplane this paper uses is the GTM, a hypothetical airplane similar to a Boeing 757 used in computer simulations of

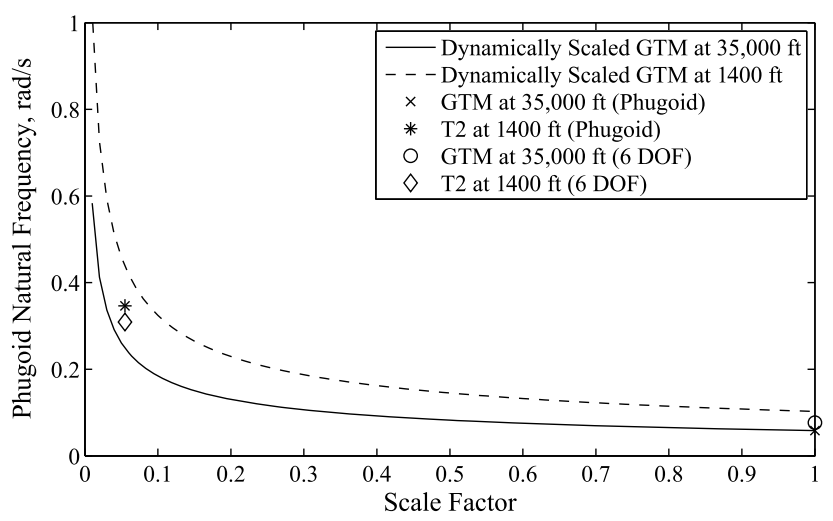

a) Linear frequency scale

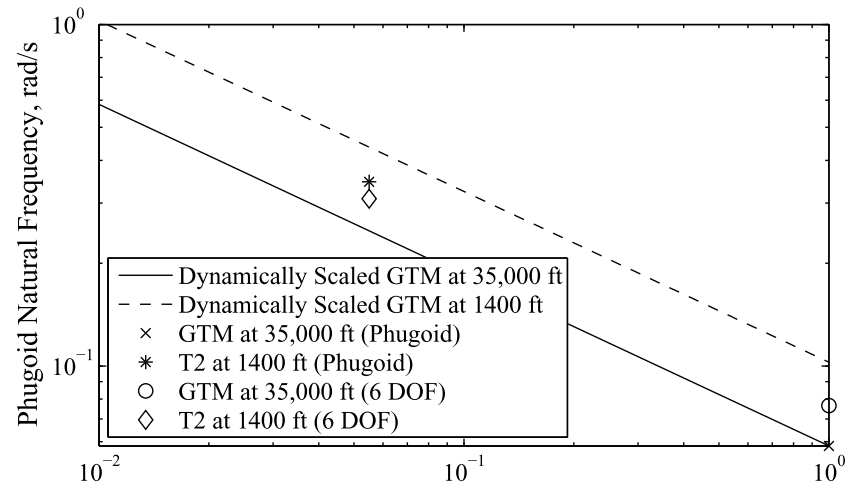

Scale Factor

b) Logarithmic frequency scale

Fig. 5 Phugoid natural frequency versus length scale factor. transport aircraft [43]. The reference condition for the chosen parameters is steady level longitudinal flight at Mach 0.8 and an altitude of $35,000 \mathrm{ft}$. The second NASA airplane this paper uses is the T2 subscale jet transport, a 5.5\% dynamically scaled model of the GTM in operational use by NASA. Hence, $N=0.05$ in Eq. (19a) when the GTM is the full-scale aircraft and the T2 is the model. Jordan et al. [23] describe the design and construction of the T2. Most of the needed aircraft parameters for the T2 are tabulated in Morelli and Cunningham [44] and correspond to steady level longitudinal flight at roughly $132 \mathrm{ft} / \mathrm{s}$ and an altitude of $1400 \mathrm{ft}$.

\section{A. Figure Format}

Figures 5-7 show numerical examples of the dynamic scaling of different performance variables described in Sec. IV. In each of the figures, the values of the variables investigated are plotted by dynamically scaling the value for the GTM in steady level

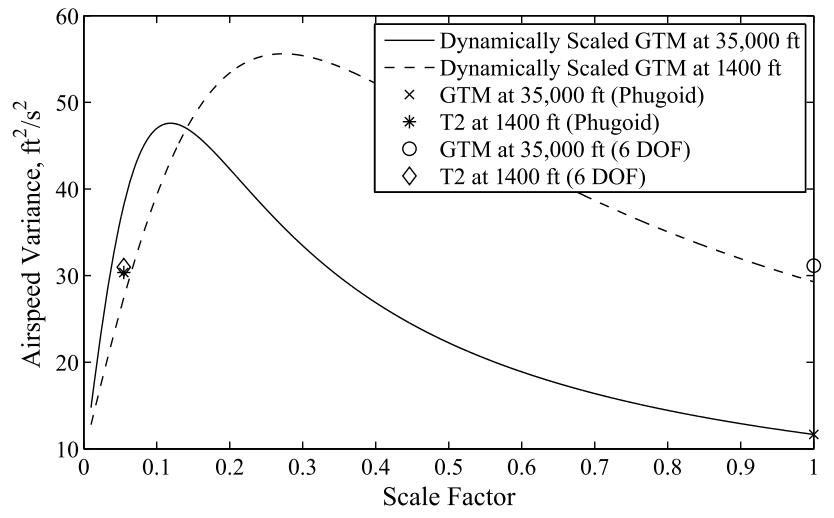

Fig. 6 Airspeed variance vs scale factor.

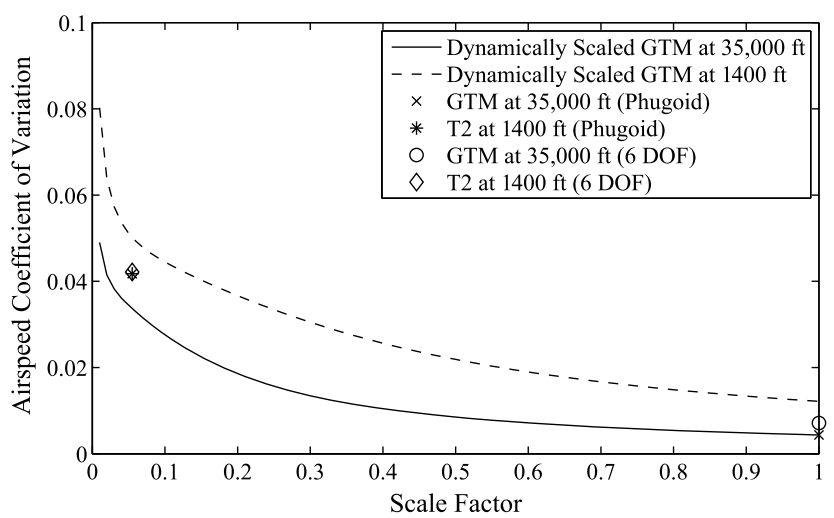

a) Linear scale

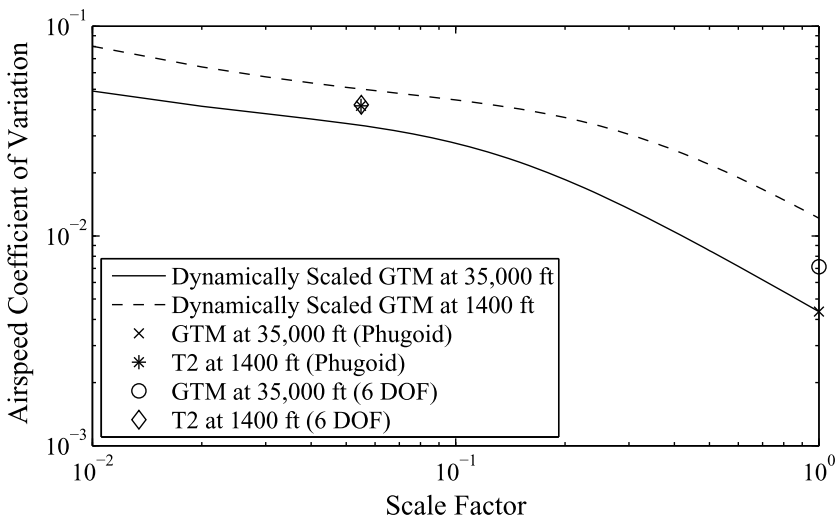

b) Logarithmic scale

Fig. 7 Coefficient of variation of the airspeed vs length scale factor. 
longitudinal flight in moderate turbulence at two altitudes using the scaling laws in Eqs. (19a-19f). The two altitudes chosen are 35,000 and $1400 \mathrm{ft}$, typical cruise altitudes of the GTM and T2, respectively. The length scale factor $N$ is the wingspan relative to the GTM's wingspan.

A choice of Mach 0.8 for the GTM at $1400 \mathrm{ft}$ is neither practical nor a good comparison to the cruising T2. For the curve corresponding to $1400 \mathrm{ft}$, the GTM airspeed has been adjusted as follows. Assume that Mach 0.8 at 35,000 ft, or $782 \mathrm{ft} / \mathrm{s}$, corresponds to the airspeed for minimum thrust $v_{\mathrm{mt}}$ for the GTM. According to McClamroch [38], the airspeed for minimum thrust in a jet aircraft is also the airspeed that minimizes the rate of fuel consumption and only depends on altitude via the air density. They are related as $v_{\mathrm{mt}}(a) \propto \rho^{-1 / 2}(a)$, in which $a$ is again the altitude. Thus, the airspeed for minimum thrust at $1400 \mathrm{ft}$ is related to the air densities at 1400 and $35,000 \mathrm{ft}$ and the airspeed for minimum thrust at $35,000 \mathrm{ft}$ as

$$
v_{\mathrm{mt}}(1400 \mathrm{ft})=\sqrt{\frac{\rho(35,000 \mathrm{ft})}{\rho(1400 \mathrm{ft})}} v_{\mathrm{mt}}(35,000 \mathrm{ft})
$$

Carrying out the computation, the GTM's airspeed for minimum thrust at $1400 \mathrm{ft}$ is $446 \mathrm{ft} / \mathrm{s}$. This airspeed is used as the reference airspeed for the GTM in the curve showing dynamic scaling at $1400 \mathrm{ft}$.

Along with the dynamic scaling curves, points are marked for the values of the GTM at $35,000 \mathrm{ft}$, and the T2 at $1400 \mathrm{ft}$. The dynamic scaling curves are plotted using the analytical solutions from Sec. IV, whereas the points for the GTM and T2 are computed by forming the respective state-space models and using a numerical Lyapunov solver. Two points are marked for each aircraft, one computed using the phugoid approximation of Eq. (34), and one computed using the full linearized model of Eq. (14).

\section{B. Scaling of the Phugoid Mode}

Equations (32) and (33) give scaling laws for the phugoid natural frequency and damping ratio, respectively. Figure 5 plots the phugoid natural frequency $\omega_{n p}$ vs the scale factor $N$ on both linear and logarithmic scales. The curves show how the GTM phugoid natural frequency scales dynamically based on Eq. (32) at 35,000 and $1400 \mathrm{ft}$. Because the phugoid natural frequency is related to the scale factor through a power law, the curves are straight lines when plotted on the logarithmic scale. The points marked show good agreement between the phugoid approximation and the full linearization's phugoid natural frequency. The T2's phugoid approximation and full linearization also have similar phugoid natural frequencies. The T2's phugoid natural frequency is below that predicted by dynamically scaling the GTM, with the T2 phugoid natural frequency about $15 \%$ below the predicted value. However, it is well above the GTM's phugoid natural frequency at $35,000 \mathrm{ft}$. Any error must come from the difference in airspeed or coefficients of lift and drag, because the other airplane parameters match well, and the altitude and turbulence parameters are the same for both.

Equation (33) shows that the phugoid damping ratio only depends on the length scale factor through the air density. Without a model of how the air density varies with length scale factor, the phugoid damping ratio cannot be plotted vs the length scale factor. Instead, Table 1 shows the phugoid damping ratio for the GTM and T2

Table 1 Phugoid eigenvalue, damping ratio, and natural frequency for the GTM and $\mathrm{T2}^{\mathrm{a}}$

\begin{tabular}{ccccc}
\hline \hline Airplane & Model & Eigenvalue & $\zeta_{p}$ & $\omega_{n p}, \mathrm{rad} / \mathrm{s}$ \\
\hline GTM & Phugoid & $-0.00257 \pm 0.0583 i$ & 0.0440 & 0.0583 \\
GTM & 6 DOF & $-0.00185 \pm 0.0766 i$ & 0.0241 & 0.0766 \\
T2 & Phugoid & $-0.0193 \pm 0.346 i$ & 0.0558 & 0.346 \\
T2 & 6 DOF & $-0.0283 \pm 0.307 i$ & 0.0918 & 0.309 \\
\hline \hline
\end{tabular}

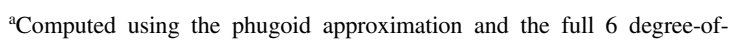
freedom (DOF) linearization. computed using the phugoid approximation and the full linearization. The GTM and T2 phugoid damping ratios are all of the same order of magnitude, with the phugoid approximations matching best. The natural frequency values in Table $\underline{1}$ correspond to the four points marked in Fig. $\underline{5}$.

\section{Scaling of the Airspeed Variance}

In Eq. (35), the variance of the airspeed depends on the airplane length scale factor through $\kappa$. Figure $\underline{6}$ shows how $\sigma_{V}^{2}$ varies with the airplane length scale factor. At both altitudes, $\sigma_{V}^{2}$ is small for small and large airplanes and has a peak in between. The scaled GTM, the phugoid approximation of the T2, and the full linearization of the T2 are all close in variance. However, the difference for the GTM between the airspeed variance in the phugoid approximation and the full linearization is substantial. Validating these dynamic scaling curves from the phugoid model against the full linearization is left as future work.

To get a better sense of the magnitude of these variances, they should be normalized. A common way to measure uncertainty in a random variable is to compute its coefficient of variation, the standard deviation divided by the mean. Because Eq. (34) is an LTI system driven by zero-mean white noise, the airspeed perturbations will also have zero mean. The mean airspeed will therefore equal the reference airspeed of the linearization.

Scaling Law 3: Airspeed Coefficient of Variation: The coefficient of variation for the airspeed is $\sigma_{V} / V$. Figure $\underline{7}$ plots the airspeed coefficient of variation vs the scale factor.

Figure 7 shows that, after normalizing by the airspeed, the uncertainty in the airspeed decreases monotonically with increasing airplane size. The figure also shows that the disagreement in airspeed variance between the phugoid approximation and the full linearization lessens after normalization. Figure $\underline{7 b}$ plots the relationship on a logarithmic scale. The airspeed variance in the numerator of the coefficient of variation scales as shown in Fig. 6, whereas the airspeed in the denominator scales as the power law in Eq. $(\underline{19 \mathrm{e}}), V \propto N^{1 / 2}$. The result is that the airspeed coefficient of variation scaling law is not a power law and not a straight line on the logarithmic scale. It exhibits the airspeed's strong dependence on the length scale factor, but is adjusted because of the dependence of $\sigma_{V}$ on the length scale factor.

The T2's coefficient of variation from the phugoid approximation is about $16 \%$ below the value for the scaled GTM but is well above the GTM's coefficient of variation at 35,000 ft. A later example shows better agreement when the airspeed of the scaled GTM is used for the $\mathrm{T} 2$ instead of the airspeed from the parameter source.

To consider how the dynamically scaled GTM compares to the other types of airplanes, consider again Fig. 1 from the Introduction, variations of which are presented in Fig. 8 . Figure 8 shows values for the airspeed variance and coefficient of variation for a variety of aircraft, all computed at $1400 \mathrm{ft}$ using the phugoid approximation. The solid curve represents the dynamically scaled, analytically determined GTM value. The dashed curve represents the dynamically scaled, analytically determined C-5A value. The numerically determined GTM and C-5A values are marked with the symbol $\times$ on the curves. All of the other plotted points correspond to numerically determined values for the indicated airplanes. The coefficient of variation is plotted on both linear and logarithmic scales. The dash-dot line corresponds to a least-squares fit of the various coefficients of variation to a power law.

Both the airspeed variance and coefficient of variation depend on the reference airspeed. The speed scaling law in Eq. (19e) suggests a simple relationship between airspeeds at different airplane sizes. In reality, aircraft can operate at a wide range of airspeeds, and design considerations other than length scale, such as weight and engine performance, play an important role in determining airspeed.

Compare, for example, the Navion general aviation aircraft and the A-4D fighter. The Navion's wingspan is $33.4 \mathrm{ft}$, and the A-4D's is $27.5 \mathrm{ft}$. We estimate that, at an altitude of $1400 \mathrm{ft}$, the Navion's airspeed for minimum fuel consumption is $100 \mathrm{ft} / \mathrm{s}$, and the A-4D's is $402 \mathrm{ft} / \mathrm{s}$. Scaling the GTM down to the size of the A-4D, we expect an airspeed for minimum fuel consumption of $209 \mathrm{ft} / \mathrm{s}$. To eliminate 


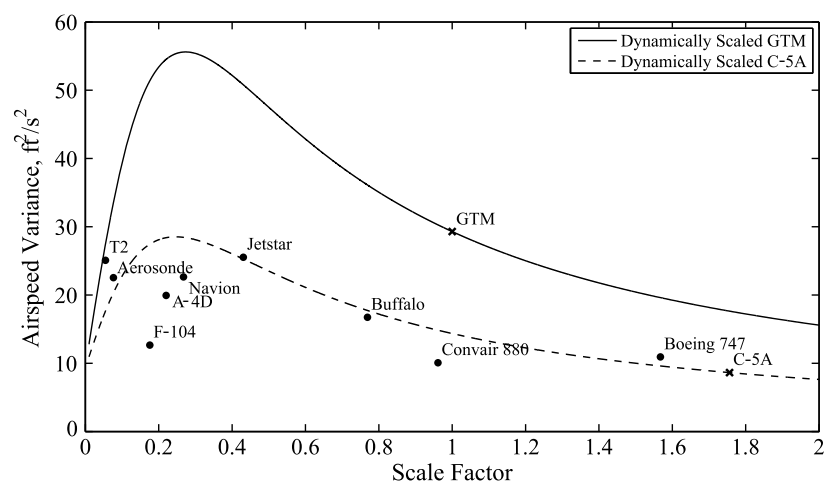

a) Airspeed variance

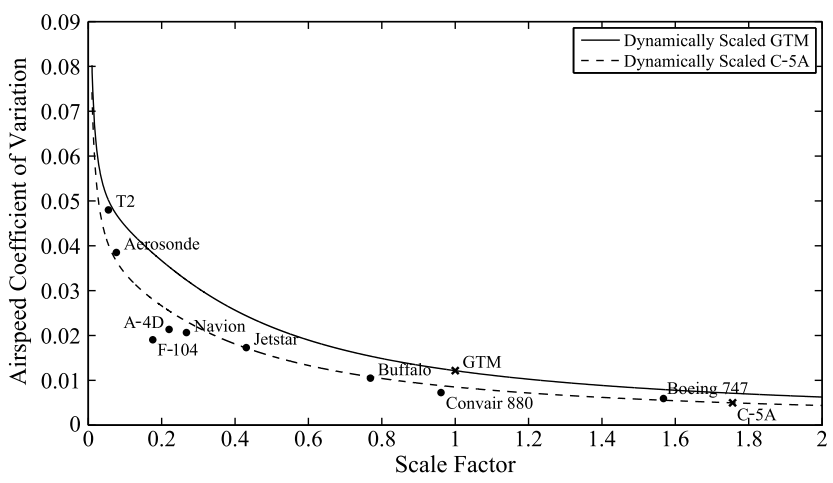

b) Airspeed coefficient of variation, Linear scale

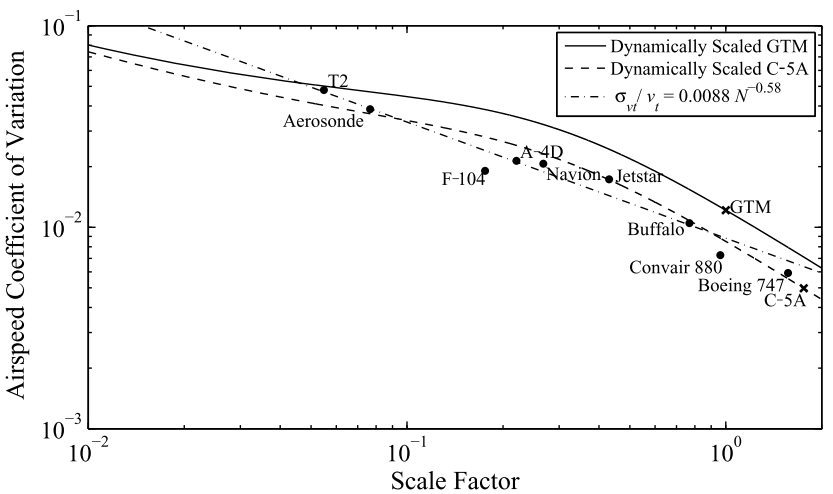

c) Airspeed coefficient of variation, Logarithmic scale

Fig. 8 Airspeed variance and coefficient of variation versus wingspan scale factor for the phugoid approximation of various airplanes.

this variability, the scaled GTM's airspeed is used for all of the airplanes in Fig. 8. The T2's airspeed is also adjusted in this manner, improving agreement between the T2 and the scaled GTM compared to the previous examples. Airplane weight exhibits similar variability, but the figures do not account for deviations of airplane weight from the scaling laws.

In Fig. , the length scale factor again corresponds to the relative wingspan. While studying the figures, note the following:

1) The T2 matches the scaled GTM's curves well, better than in the previous examples, in which the airspeed from the source of the T2's parameters was used.

2) Neither fighter matches the scaled transports well.

3) Both the GTM and the T2 appear to be unusually sensitive to gusts for aircraft at their wingspans.

4) The Navion's airspeed variance is lower than the scaled GTM's and the scaled C-5A's.

5) The rest of the aircraft follow the trend of the dynamically scaled C-5A.

The poor agreement between the scaling law and the fighters is expected for the reasons described earlier related to performance of the fighters compared to performance of transport aircraft of their size. Moreover, scaling by wingspan assumes that the various aircraft have geometrically similar planforms. This assumption is reasonable among the transports but not between the transports and the fighters. The fighters, the Navion, and the Convair 880 have less airspeed variation than the scaled C-5A, whereas the GTM and the T2 have more; all of these deviations from the scaled C-5A can be explained by comparing the airplanes' phugoid damping ratios.

Table B1 in Appendix B lists, for each airplane in Fig. 8, the values of $\kappa$, the $\overline{\text { GTM} M ' s ~ s c a l e d ~ v a l u e ~ o f ~} \kappa$, and $\zeta_{p}$. While the scaled GTM's $\kappa$ does not predict the values of $\kappa$ for the other airplanes well, the trends match. As for the phugoid damping ratio, according to Eq. (33), it should be the same for all airplanes at fixed altitude, but it clearly varies between the different aircraft types. For every airplane in Fig. 8, the damping ratio relative to the C-5A's determines whether the airspeed variance and coefficient of variation are above or below the C-5A's dynamically scaled value. Moreover, the GTM and the F104 , which have the lowest and highest damping ratios, respectively, are the biggest outliers. Thus, the dependence of the airspeed variance on $\zeta_{p}$ and the difference between the various airplanes' values for $\zeta_{p}$ limit the accuracy of the scaling laws.

As before, the derived GTM and C-5A airspeed coefficients of variation do not scale as power laws. This is caused by the non-power law scaling of the airspeed variance and is evident in Fig. $8 \mathrm{c}$, in which their curves are not straight lines. The straight line in Fig. $\overline{8 c}$, which is the least-squares fit of the various coefficients of variation to a power law, shows that $\sigma_{V} / V \propto N^{-0.58}$.

That the exponent is close to $-1 / 2$ is not surprising, since $V \propto N^{1 / 2}$. As mentioned earlier, the coefficient of variation exhibits the scaling of the airspeed adjusted for the scaling of the airspeed variance. The power law fit is a good match for the smallest aircraft, and slightly overpredicts the coefficients of variation of the largest aircraft, which is the case in the upcoming examples as well.

\section{Other Length-Scale Factors}

Relative wingspan is one of many potential length scale factors to use for these scaling laws. This section compares scaling based on relative wingspan with scaling based on relative wing loading and relative weight.

In some applications, the wing loading $\mathrm{mg} / \mathrm{S}$ is the preferred length scale factor, as in Tennekes's great flight diagram [42]. In fact, the wing loading is very relevant to this problem because Eqs. (30) and (31) show that the phugoid natural frequency and damping ratio can be written in terms of wing loading. While wing loading is not a length, the scaling laws in Eqs. (19b) and (19c) show that it should scale proportional to length.

Figure 9 shows the same scaling laws as Fig. 8, except with the length scale factor and the chosen airspeeds corresponding to relative wing loading. Note that, even though the T2 is designed as a $5.5 \%$ dynamically scaled model of the GTM, its relative wing loading actually corresponds to a length scale factor of $8.7 \%$. Also note that the T2's reference airspeed from the source of the parameters [44] and the scaled GTM's airspeed based on relative wing loading differ by less than $3 \%$. Therefore, the T2's airspeed variance and coefficient of variation do not change significantly from the examples earlier that use the airspeed given in the T2 parameters' source.

From the plots, the trends shown in the previous examples are apparent, with the F-104 and the GTM still being outliers and the smallest airplanes having the largest airspeed coefficients of variation. With the exception of the F-104, all of the aircraft's variances and coefficients of variation are bounded by those of the dynamically scaled GTM and C-5A. However, neither the dynamically scaled GTM nor the dynamically scaled C-5A captures the precise values of the other aircraft, especially for the variance, when compared with using the relative wingspan as the length scale factor. Once again, the power law fit of the coefficient of variation leaves the C-5A as an outlier and has an exponent close to $-1 / 2$.

Another preferred length scale factor is the cube root of the airplane mass, $\sqrt[3]{m}$, as in Liu [21]. As with wing loading, the cube root of the mass is not a length, but scales proportional to length, as 
seen in Eq. (19c). Also, like the wing loading, the mass features prominently in the phugoid approximation, suggesting it should also be a more relevant length scale than wingspan. Scaling with the relative mass also weakens the assumption of similar planforms among the aircraft.

Figure 10 shows the same scaling laws as Figs. 8 and $\underline{9}$, except with the length scale factor and the chosen airspeeds corresponding to the cube root of the relative mass. Using this length scale factor, the trends and major outliers remain the same. For the airspeed variance, the scaled C-5A and the other aircraft show better agreement than when scaled by wing loading, but relative wingspan still provides the best fit and agreement related to the damping ratio. As with the other two choices of scale factor, the power law fit of the coefficient of variation leaves the $\mathrm{C}-5 \mathrm{~A}$ as an outlier and has an exponent close to $-1 / 2$. Scaling based on relative weight results in better agreement between the airspeed coefficient of variation scaling law and numerical solution compared to scaling based on relative wing loading, but it is not clear if it is an improvement over scaling based on relative wingspan. Overall, the scaling laws in this paper are good predictors of the statistics of airspeed variations due to turbulence in

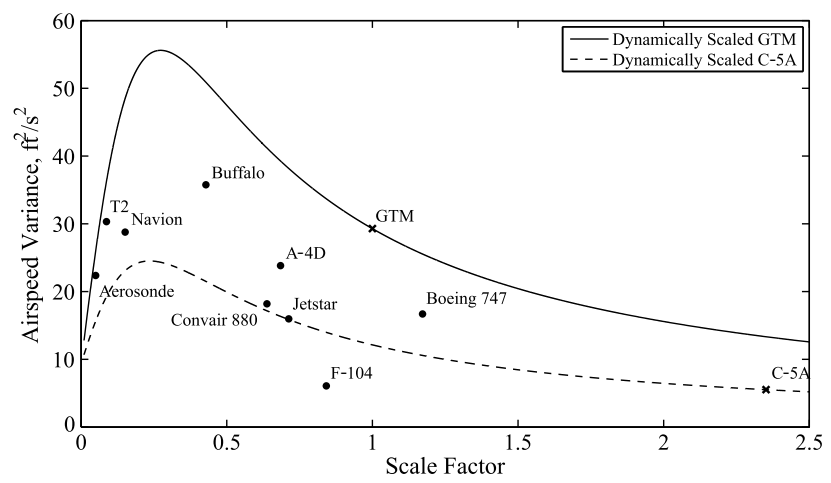

a) Airspeed variance

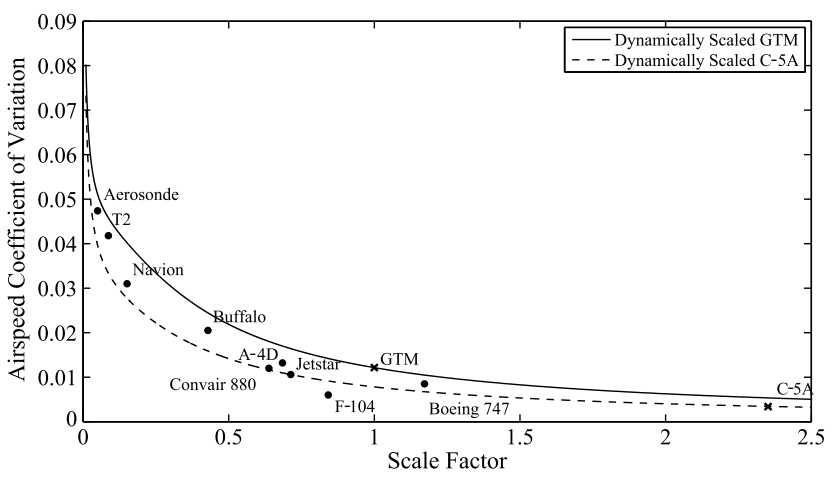

b) Airspeed coefficient of variation, Linear scale

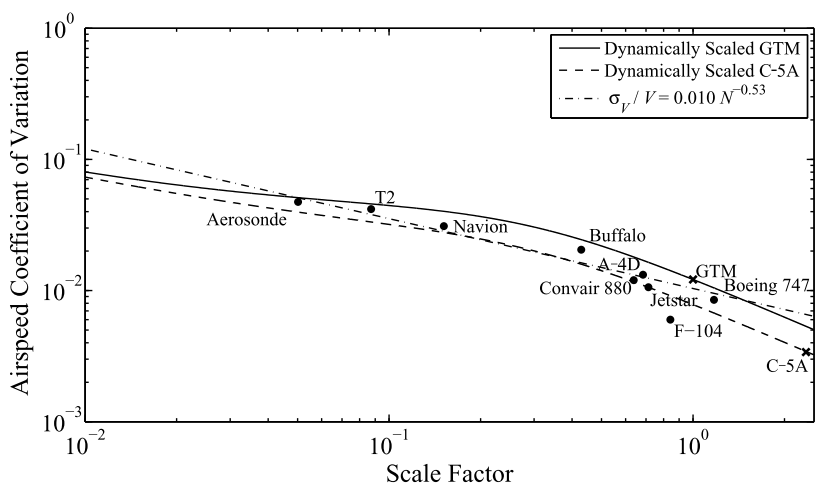

c) Airspeed coefficient of variation, Logarithmic scale

Fig. 9 Airspeed variance and coefficient of variation versus wing loading scale factor for the phugoid approximation of various airplanes.

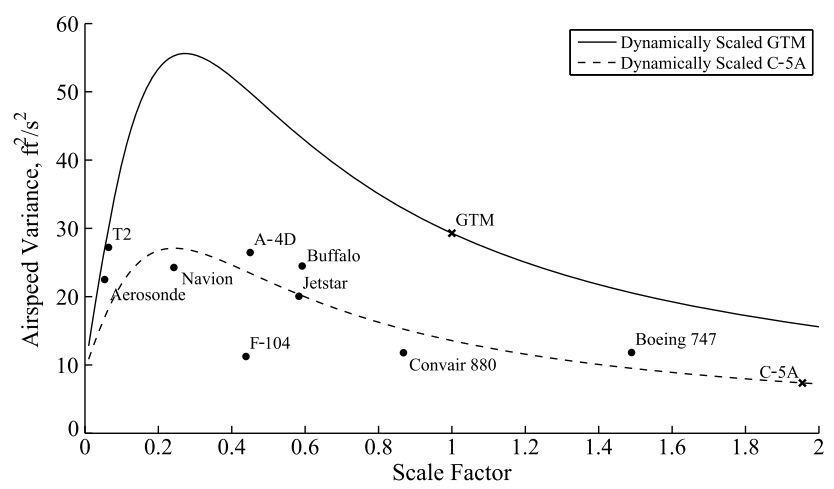

a) Airspeed variance

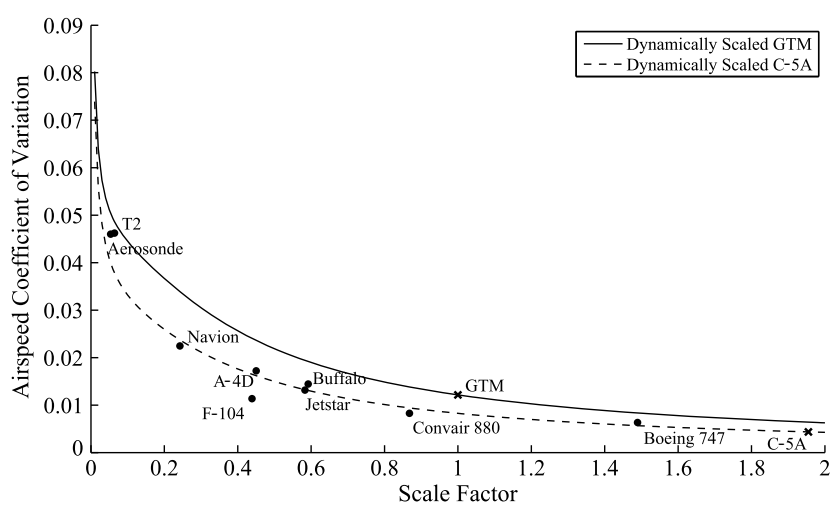

b) Airspeed coefficient of variation, Linear scale

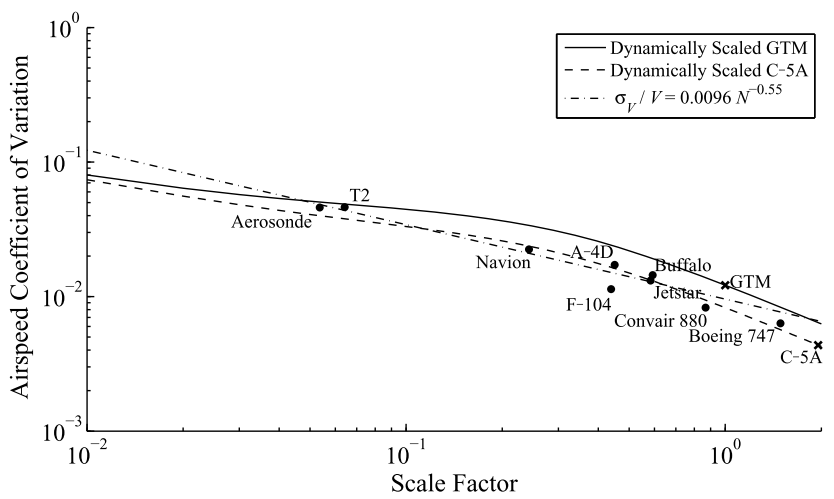

c) Airspeed coefficient of variation, Logarithmic scale

Fig. 10 Airspeed variance and coefficient of variation versus cub-rootof-the-mass scale factor for the phugoid approximation of various airplanes.

the phugoid approximation, particularly the airspeed coefficient of variation. Furthermore, the examples suggest that relative wingspan is a better measure of length scale than wing loading for this set of scaling laws.

\section{Scaling of Flight Envelopes}

The authors' previous work relied on airspeed statistics to compute safety margins for steady-flight maneuvers performed in turbulence. It also proposed flight envelopes to visualize these safety margins [8]. The authors introduced an extension of steady flight called stationary flight, flight in which the linear- and angular velocity vectors are stationary random processes. Section II notes that, in Eq. (14), the linear- and angular velocity perturbations are zero-mean, stationary, Gaussian random processes. Therefore, modeling turbulence response in this manner means that steady-flight maneuvers performed in turbulence result in stationary flight. Section II also notes that the covariance matrix of the airplane and wind linear 


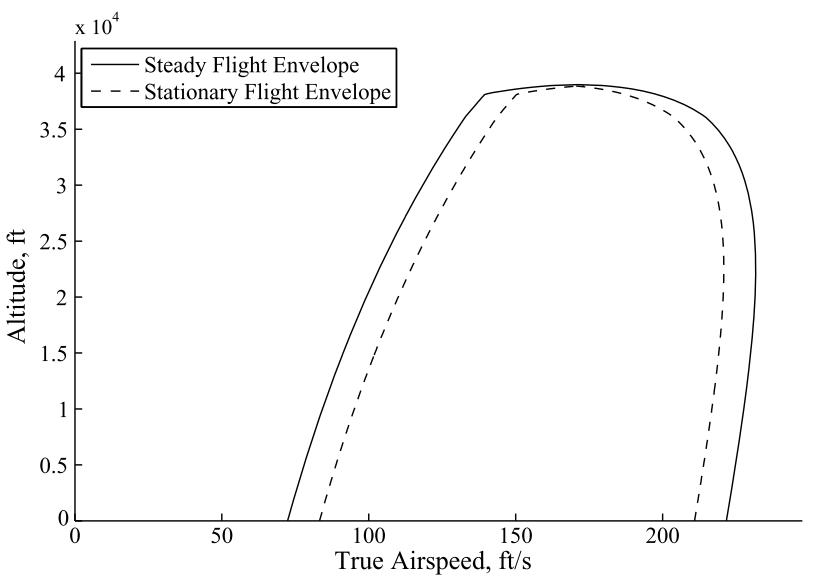

a) Navion. Adapted from [8]

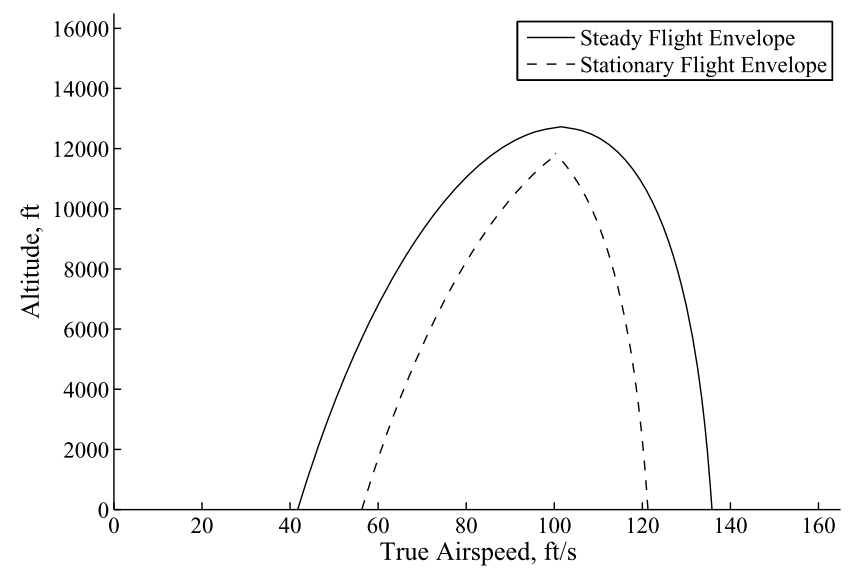

b) Aerosonde

Fig. 11 Steady and stationary flight envelopes.

velocities can be computed using a Lyapunov equation, so the variance of the true airspeed can be computed.

Staying within the steady flight envelope improves safety of flight because airplanes are generally designed to fly stably or stabilizably when flying steadily, and may be prone to loss of control outside of the steady flight envelope. In the previous work, the authors proposed shifting the steady flight envelope boundaries inward proportional to the standard deviation of the airspeed. The authors call the resulting envelope a stationary flight envelope. For a shift of three standard deviations and a Gaussian true airspeed distribution, flying within the stationary flight envelope guarantees that $99.87 \%$ of true airspeed fluctuations remain within the steady flight envelope.

For level longitudinal flight using the full linearization of Sec. II, linearizing the Navion around a flight state of $102 \mathrm{ft} / \mathrm{s}$ at an altitude of $16,500 \mathrm{ft}$, and the Aerosonde around a flight state of $83 \mathrm{ft} / \mathrm{s}$ at an altitude of $4950 \mathrm{ft}$ gives true airspeed coefficients of variation of 3.8 and $5.8 \%$, respectively. Each of these flight states is near the stall speed for the respective airplane. Figure 11 shows the level longitudinal steady and stationary flight envelopes for the two airplanes. The Navion's envelope is reproduced from the authors' previous work [8]. For both airplanes, flight states close to the flight envelope boundaries become prone to flight envelope excursions in turbulence, with excursions across the stall boundary being the most dangerous. However, the adjustment to the steady flight envelope to form the stationary flight envelope results in a more substantial envelope reduction for the Aerosonde than the Navion. The higher coefficient of variation and the more substantial flight envelope reduction of the Aerosonde compared to the Navion are consistent with the scaling laws of the previous section, even though the present example does not control for altitude or airspeed. The authors intend for the turbulence response models and scaling laws developed in this paper to improve understanding of airplane performance in turbulence and aid analyses such as the flight envelopes of Fig. 11 when airplane parameters are not available.

\section{Conclusions}

Using a phugoid approximation of the linearized airplane dynamic equations, this paper derives scaling laws for the phugoid mode natural frequency and damping ratio, as well as expressions for the airspeed and flight-path angle variances. It also gives numerical examples of the results of the phugoid analysis, and compares them to stability derivative models of the NASA GTM and T2. The numerical results include plots showing how the airspeed coefficient of variation scales with airplane size and compare the $\mathrm{T} 2$ and dynamically scaled GTM to a variety of other airplanes.

Using two metrics, the results show that small airplanes are more susceptible to turbulence than large airplanes. First, the scaling of the phugoid natural frequency shows that the dynamics of large airplanes filter out more high frequency gusts than the dynamics of small airplanes. Second, the coefficient of variation for airspeed decreases as airplanes get larger, showing that variations in airspeed due to turbulence, when expressed as a fraction of the nominal airspeed, are smaller for large airplanes. These results, which from a qualitative standpoint are intuitive reemphasize the need to make autopilots on small airplanes robust to turbulence and provide a quantitative basis to compare gust responses of airplanes of different sizes. As smallscale aircraft become more prevalent in military and commercial applications, these scaling laws can serve as useful tools to predict and improve airplane performance in gusts.

\section{Appendix A: Parameters for the Numerical Example}

This paper's numerical examples use models of the NASA Generic Transport Model (GTM) and T2 transport aircraft. The GTM parameters primarily come from a GTM simulator developed at NASA Ames Research Center. The model is described in an internal NASA report [43] and applied in Nguyen et al. [45]. The simulator contains tables of stability derivatives parameterized by Mach number and angle of attack. Nguyen et al. $[45,46]$ use the GTM to study damaged, asymmetric transport aircraft, but this paper uses the parameters for the intact GTM. The parameters selected correspond to Mach 0.8 with an angle of attack of $2 \mathrm{deg}$. The simulator does not specify an altitude for the parameters, so the authors assume they are valid at 35,000 ft. The GTM is similar to the Boeing 757, so this Mach number and altitude are chosen based on Boeing 757 specifications posted on the American Airlines website [47], and the angle of attack is chosen based on a Boeing article about commercial-jet angles of attack [48].

Stability derivatives for the NASA T2 Subscale Jet Transport are given in the appendix of Morelli and Cunningham [44]. According to the flight test results, the stability derivatives correspond to a nominal flight condition of level longitudinal flight with an airspeed of roughly $132 \mathrm{~m} / \mathrm{s}$ at $1400 \mathrm{ft}$ with an angle of attack of $4.5 \mathrm{deg}$. Morelli and Cunningham [44] do not give values for the reference coefficients of lift or drag, $C_{L_{0}}$ and $C_{D_{0}}$. These coefficients are estimated for this paper using standard equations for steady level longitudinal flight performance given in McClamroch [38]. Specifically, the following two expressions for the coefficient of lift are equated and solved for $C_{L_{0}}$ :

$$
\begin{gathered}
C_{L}=\frac{2 m g}{\rho S V^{2}} \\
C_{L}=C_{L_{0}}+C_{L_{\alpha}} \alpha
\end{gathered}
$$

For the reference drag coefficient, the GTM's airspeed for minimum thrust at $1400 \mathrm{ft}$ computed in Eq. (43) is scaled using Eq. (19e) to the corresponding $\mathrm{T} 2$ airspeed for minimum thrust $v_{\mathrm{mt}}$. Carrying out the computation, $v_{\mathrm{mt}}=104 \mathrm{ft} / \mathrm{s}$ for the $\mathrm{T} 2$ at $1400 \mathrm{ft}$ altitude. This airspeed is related to the reference drag coefficient as 


$$
v_{\mathrm{mt}}=\sqrt{\frac{2 m g}{\rho \sqrt{C_{D_{0}} \pi e S b^{2}}}}
$$

These relationships lead to the values

$$
C_{L_{0}}=0.129, \quad C_{D_{0}}=0.0252
$$

Figures 1 and $8-10$ include results for several other aircraft. Beard and McLain [49] provide the Aerosonde model. Teper [50] provides the F-104, A- $\overline{4 D}$, and Ryan Navion models. Heffley and Jewell [51] provide the Lockheed Jetstar, Convair 880 , Boeing 747, and C- $\overline{5 A}$ models. MacDonald et al. [52] provide the de Havilland Canada Buffalo model. The Aerosonde model does not give a reference flight state. A reference airspeed of $60 \mathrm{kt}$ is chosen as its cruise airspeed based on the manufacturer's specifications [53]. The C-5A model used is for sea level at Mach 0.45. The Buffalo model used is for sea level at $215 \mathrm{ft} / \mathrm{s}$. The remaining aircraft models are reproduced in Appendix B of Nelson [11], and this paper uses the sea level flight states listed in that text. For all of these models, except the Aerosonde and the Buffalo, $C_{L}, C_{D}, C_{L_{\alpha}}$, and $\alpha$ are given for the reference flight state of the model, but this paper requires $C_{D_{0}}$. $C_{D_{0}}$ is computed for each aircraft from the drag polar in McClamroch [38]:

$$
C_{D}=C_{D_{0}}+\frac{S C_{L}^{2}}{\pi e b^{2}}
$$

For all of the aircraft, the analysis required a value for the Oswald efficiency factor $e$ to compute the coefficient for induced drag in the drag polar. This parameter is rarely provided, although the Buffalo model specifies 0.75 . This paper uses the value 0.8 for all of the other aircraft. For the air density, this paper uses the U.S. Standard Atmosphere calculator provided in McClamroch [38]. For the Dryden gusts, this paper adheres to the MIL-STD-1797A specifications [31] and the filters given in the MATLAB documentation [39]. The turbulence intensity and scale length vary with altitude. Because the high-altitude turbulence intensity must be read off of a plot, the authors used some discretion in choosing values. For moderate turbulence, the authors use $\sigma_{u}=5 \mathrm{ft} / \mathrm{s}$ at $35,000 \mathrm{ft}$ and $\sigma_{u}=9 \mathrm{ft} / \mathrm{s}$ at $2000 \mathrm{ft}$. The U.S. Standard Atmosphere model uses altitude above mean sea level, whereas the turbulence models use altitude above ground level. For convenience, this paper assumes that the ground is at sea level.

\section{Appendix B: Relevance of Unsteady Effects}

Hoblit shows that, in the short period approximation, the dimensionless parameter $\bar{c} / \delta_{\mathrm{sp}}$ is a measure of the importance of unsteady effects in turbulence response [30]. $\delta_{\mathrm{sp}}$ is defined in Eq. (42) for the short period approximation. Here, the authors posit a phugoid analog of $\delta_{\mathrm{sp}}$ :

$$
\delta_{p} \triangleq \frac{2 m}{\rho S C_{L}}
$$

based on the way the lift coefficient enters the two approximations.

Table B1 shows the values of the relevant nondimensional parameters for the 11 airplane models used in Fig. 8 . The last column of Table B1 lists the values for $\bar{c} / \delta_{p}$. Hoblit [30] shows that, for values of $\bar{c} / \delta_{\mathrm{sp}} \leq 0.010$, the gust response does not change significantly due to unsteady effects. Because each of the airplanes listed in Table B1 has a value for $\bar{c} / \delta_{p}$ that is less than 0.010 , unsteady effects are not expected for any combination of airplane and flight state considered in this paper.

\section{Acknowledgments}

This work was supported in part by the U.S. Air Force under grant number FA 8650-07-2-3744 and by the U.S. Army Research Office
Table B1 Dimensionless parameters for the airplanes in Fig. $\underline{8}$

\begin{tabular}{ccclc}
\hline \hline Airplane & $\kappa$ & Scaled GTM's $\kappa$ & $\zeta_{p}$ & $\bar{c} / \delta_{p}$ \\
\hline T2 & 5.5 & 1.7 & 0.050 & 0.0027 \\
Aerosonde & 3.9 & 1.2 & 0.078 & 0.0013 \\
F-104 & 1.7 & 0.52 & 0.30 & 0.0088 \\
A-4D & 1.4 & 0.41 & 0.14 & 0.0080 \\
Navion & 1.1 & 0.34 & 0.12 & 0.0035 \\
Jetstar & 0.70 & 0.21 & 0.091 & 0.0041 \\
Buffalo & 0.39 & 0.12 & 0.10 & 0.0021 \\
Convair 880 & 0.31 & 0.094 & 0.14 & 0.0032 \\
GTM & 0.30 & 0.30 & 0.044 & 0.0027 \\
Boeing 747 & 0.19 & 0.058 & 0.080 & 0.0028 \\
C-5A & 0.17 & 0.052 & 0.092 & 0.0028 \\
\hline \hline
\end{tabular}

through the National Defense Science and Engineering Graduate Fellowship Program.

\section{References}

[1] Federal Aviation Administration, "Review of Aviation Accidents Involving Weather Turbulence in the United States," National Aviation Safety Data Analysis Center, Washington, D.C., 2004.

[2] Federal Aviation Administration, "Air Operators Utilization Reports," Aviation Data Systems Branch, Washington, D.C., 2001.

[3] Federal Aviation Administration, "General Aviation and Air Taxi Activity (GAATA) Survey CY2001," Statistics and Forecasts Branch, Washington, D.C., 2001.

[4] "Unmanned Aircraft Systems: Comprehensive Planning and a ResultsOriented Training Strategy Are Needed to Support Growing Inventories," U.S. Government Accountability Office, Washington, D.C., GAO-10-331, March 2010.

[5] U.S. Dept. of Defense, "Unmanned Aircraft Systems Roadmap 2005 2030," Office of the Secretary of Defense, Washington, D.C., 2005.

[6] Federal Aviation Administration, "Pilot/Controller Glossary," Federal Aviation Administration, Washington, D.C., 2011.

[7] Richardson, J. R., Atkins, E. M., Kabamba, P. T., and Girard, A. R., "Envelopes for Flight Through Stochastic Gusts," AIAA Paper 20116213, Aug. 2011.

[8] Richardson, J. R., Atkins, E. M., Kabamba, P. T., and Girard, A. R., "Envelopes for Flight Through Stochastic Gusts," Journal of Guidance, Control, and Dynamics, Vol. 36, No. 5, 2013, pp. 1464-1476. doi:10.2514/1.57849

[9] Richardson, J. R., "Quantifying and Scaling Airplane Performance in Turbulence,” Ph.D. Thesis, Univ. of Michigan, Ann Arbor, MI, 2013.

[10] Etkin, B., and Reid, L. D., Dynamics of Flight: Stability and Control, 3rd ed., Wiley, New York, 1996, pp. 259-302, Chap. 8.

[11] Nelson, R. C., Flight Stability and Automatic Control, 2nd ed., WCB/ McGraw-Hill, Boston, 1998, pp. 101-127, Chap. 3.

[12] Roskam, J., Airplane Flight Dynamics and Automatic Flight Controls, Vol. 1, Design, Analysis, and Research Corp., Lawrence, KS, 2003, pp. 53-236, Chaps. 3-4.

[13] Stengel, R. F., Flight Dynamics, Princeton Univ. Press, Princeton, NJ, 2004, pp. 457-473, Chap. 5.

[14] Etkin, B., Dynamics of Atmospheric Flight, Dover, Mineola, NY, 2005, pp. 529-556, Chap. 13.

[15] Etkin, B., "Turbulent Wind and Its Effect on Flight," Journal of Aircraft, Vol. 18 , No. 5,1981 , pp. 327-345. doi: $10.2514 / 3.57498$

[16] Davison, D. E., Kabamba, P. T., and Meerkov, S. M., "Robustness with Respect to Disturbance Model Uncertainty: Theory and Application to Autopilot Performance Analysis," Mathematical Problems in Engineering, Vol. 6, Nos. 2-3, 2000, pp. 267-304. doi: $10.1155 \% 2$ FS1024123X00001356

[17] Mulgund, S. S., and Stengel, R. F., "Optimal Recovery from Microburst Wind Shear," Journal of Guidance, Control, and Dynamics, Vol. 16, No. 6, 1993, pp. 1010-1017. doi: $10.2514 / 3.21121$

[18] Hinton, D. A., "Forward-Look Wind-Shear Detection for Microburst Recovery," Journal of Aircraft, Vol. 29, No. 1, 1992, pp. 63-66. doi: $10.2514 / 3.46125$

[19] Burk, S. M., and Wilson, C. F., "Radio-Controlled Model Design and Testing Techniques for Stall/Spin Evaluation of General-Aviation Aircraft," SAE National Business Aircraft Meeting, Wichita, KS, Society of Automotive Engineers, Warrendale, PA, 1975. 
[20] Wolowicz, C. H., Bowman, J., James, S., and Gilbert, W. P., "Similitude Requirements and Scaling Relationships as Applied to Model Testing," NASA TP-1435, 1979.

[21] Liu, T., "Comparative Scaling of Flapping- and Fixed-Wing Flyers," AIAA Journal, Vol. 44, No. 1, 2006, pp. 24-33. doi: $10.2514 / 1.4035$

[22] Mettler, B., Tischler, M. B., and Kanade, T., "System Identification of Small-Size Unmanned Helicopter Dynamics," American Helicopter Society 55th Forum, Montreal, Quebec, American Helicopter Society Inc., Alexandria, VA, 1999.

[23] Jordan, T. L., Langford, W. M., and Hill, J. S., "Airborne Subscale Transport Aircraft Research Testbed-Aircraft Model Development," AIAA Paper 2005-6432, Aug. 2005.

[24] Shyy, W., Lian, Y., Tang, J., Viieru, D., and Liu, H., Aerodynamics of Low Reynolds Number Flyers, Cambridge Aerospace Series, Cambridge Univ. Press, Cambridge, England, U.K., 2008, pp. 14-20, 44-57, Chaps. 1-2.

[25] Gibbings, J. C., Dimensional Analysis, Springer-Verlag, London, 2011, pp. 83-93, Chap. 4.

[26] Szirtes, T., and Rózsad, P., Applied Dimensional Analysis and Modeling, 2nd ed., Elsevier/Butterworth-Heinemann, Amsterdam, 2007, pp. $463-$ 525, Chap. 17.

[27] Newman, J. N., Marine Hydrodynamics, MIT Press, Cambridge, MA, 1977, pp. 8-46, Chap. 1

[28] Ryutov, D. D., Remington, B. A., Robey, H. F., and Drake, R. P., "Magnetohydrodynamic Scaling: From Astrophysics to the Laboratory," Physics of Plasmas, Vol. 8, No. 5, 2001, pp. 1804-1816. doi:10.1063/1.1344562

[29] White, F. M., Fluid Mechanics, 5th ed., McGraw-Hill, New York, 2003, pp. 293-340, Chap. 5.

[30] Hoblit, F. M., Gust Loads on Aircraft: Concepts and Applications, AIAA Education Series, AIAA, Washington, D.C., 1988, pp. 21-68, Chap. 4.

[31] U.S. Dept. of Defense, "Flying Qualities of Piloted Aircraft," U.S. Dept. of Defense, MIL-STD-1797A, pp. 673-695, Washington, D.C., 1990, Appendix A.

[32] Dodds, C. J., and Robson, J. D., "The Description of Road Surface Roughness," Journal of Sound and Vibration, Vol. 31, No. 2, 1973, pp. $175-183$. doi:10.1016/S0022-460X(73)80373-6

[33] Bishop, R. E., and Price, W. G., Hydroelasticity of Ships, Cambridge Univ. Press, London, 1979, pp. 326-387, Chap. 11.

[34] Lloyd, A. J., Seakeeping: Ship Behaviour in Rough Weather, Ellis Horwood, Chichester, England, U.K., 1989, pp. 93-120, Chap. 4

[35] Federal Aviation Administration, "Airworthiness Standards: Transport Category Airplanes," U.S. Government Printing Office, U.S. Code of Federal Regulations, 14 CFR Pt. 25, Washington, D.C., 2011, Appendix G.
[36] Houbolt, J. C., “Atmospheric Turbulence,” AIAA Journal, Vol. 11, No. 4, 1973, pp. 421-437. doi: $10.2514 / 3.50485$

[37] Murrow, H. N., Pratt, K. G., and Houbolt, J. C., "NACA/NASA Research Related to Evolution of U.S. Gust Design Criteria," AIAA Paper 1989-1373, April 1989.

[38] McClamroch, N. H., Steady Aircraft Flight and Performance, Princeton Univ. Press, Princeton, NJ, 2011, pp. 14, 115, Chaps. 2-10.

[39] MathWorks, "Dryden Wind Turbulence Model (Continuous)," MATLAB Reference Pages, MathWorks, Natick, MA, 2010.

[40] Gajić, Z., and Qureshi, M. T. J., Lyapunov Matrix Equation in System Stability and Control: Mathematics in Science and Engineering, Academic Press, San Diego, CA, 1995, p. 62, Chap. 2.

[41] Jordan, T. L., Foster, J. V., Bailey, R. M., and Belcastro, C. M., "AirSTAR: A UAV Platform for Flight Dynamics and Control System Testing," AIAA Paper 2006-3307, June 2006.

[42] Tennekes, H., The Simple Science of Flight: From Insects to Jumbo Jets, MIT Press, Cambridge, MA, 2009, p. 15.

[43] Nguyen, N., Nelson, A., and Pulliam, T., "Damage Adaptive Control System Research Report,” Internal NASA Rept., 2006.

[44] Morelli, E. A., and Cunningham, K., "Aircraft Dynamic Modeling in Turbulence," AIAA Paper 2012-4650, Aug. 2012.

[45] Nguyen, N., Krishnakumar, K., Kaneshige, J., and Nespeca, P., "Dynamics and Adaptive Control for Stability Recovery of Damaged Asymmetric Aircraft," AIAA Paper 2006-6049, Aug. 2006.

[46] Nguyen, N., Krishnakumar, K., Kaneshige, J., and Nespeca, P., "Flight Dynamics and Hybrid Adaptive Control of Damaged Aircraft," Journal of Guidance, Control, and Dynamics, Vol. 31, No. 3, 2008, pp. 751-764. doi: $10.2514 / 1.28142$

[47] Boeing 757, American Airlines, Ft. Worth, TX, http://www.aa.com/il8n/ aboutUs/ourPlanes/boeing757.jsp, Feb. 2013.

[48] Cashman, J. E., Kelly, B. D., and Nield, B. N., "Operational Use of Angle of Attack on Modern Commercial Jet Airplanes," Aero Magazine, No. 12, 2000, pp. 10-22.

[49] Beard, R. W., and McLain, T. W., Small Unmanned Aircraft: Theory and Practice, Princeton Univ. Press, Princeton, NJ, 2012, pp. 275-276, Appendix E.

[50] Teper, G. L., “Aircraft Stability and Control Data," NASA CR-96008, 1969.

[51] Heffley, R. K., and Jewell, W. F., "Aircraft Handling Qualities Data," NASA CR-2144, 1972.

[52] MacDonald, R. A., Garelick, M., and O'Grady, J., "Linearized Mathematical Models for de Havilland Canada Buffalo and Twin Otter STOL Transports," Transportation Systems Center, DOT-TSC-FAA71-8, Cambridge, MA, 1971.

[53] Aerosonde® Mark 4.7: Redefining Expeditionary, AAI Corp., Hunt Valley, MD, 2010. 